Noname manuscript No.

(will be inserted by the editor)

\title{
A non-local cross-diffusion model of population dynamics II: Exact, approximate, and numerical traveling waves in single and multi-species populations
}

\author{
Andrew L. Krause • Robert A. Van Gorder
}

Received: date / Accepted: date

\begin{abstract}
We study traveling waves in a non-local cross-diffusion type model, where organisms move along gradients in population densities. Such models are valuable for understanding waves of migration and invasion, and how directed motion can impact such scenarios. In this paper we demonstrate the emergence of traveling wave solutions, studying properties of both planar and radial wavefronts in one and two species variants of the model. We compute exact traveling wave solutions in the purely diffusive case, and then perturb these solutions to analytically capture the influence directed motion has on these exact solutions. Using linear stability analysis, we find that the minimum wavespeeds correspond to the purely diffusive case, but numerical simulations suggest that advection can in general increase or decrease the observed wavespeed substantially, which allows a single species to more rapidly move into unoccupied resource rich spatial regions, or modify the speed of an invasion for two populations. We also find interesting effects from the non-local interactions in the model, suggesting that single species invasions can be enhanced with stronger non-locality, but that invasion of a competitive species may be slowed due to this non-local effect. Finally we simulate pattern formation behind waves of invasion, showing that directed motion can have substantial impacts not only on wave speed but also on the existence and structure of emergent patterns, as predicted in the first part of our study (Taylor et al, 2020).
\end{abstract}

Keywords aggregation · directed motion · traveling waves

\section{Introduction}

Since Fisher's famous work on waves of advantageous genes in a population (Fisher, 1937), the emergence of wave solutions in reaction-diffusion models in and beyond population ecology has been well studied (Ablowitz and Zeppetella, 1979; Dunbar, 1983, 1984; Miller, 1997; Hosono, 1998; Grindrod, 1991; Hosono, 1998; Murray, 2003; Volpert and Petrovskii, 2009). Such solutions have been used to investigate invasion fronts (Lewis et al, 2016) of species which locally out-compete their native competitors, such as in the invasion of grey squirrels in Britain (Okubo et al, 1989; White et al, 2015), or in studying the invasive properties of cancer cells (Sherratt, 1993; Strobl et al, 2020). Many other ecologically relevant scenarios require the knowledge of how different kinds of dispersal mechanisms lead to different spreading speeds of a population, and reaction-diffusion systems have been a central model of study in such spreading processes.

Traveling wave studies have been extended to a variety of other dispersal mechanisms, especially those including density-dependent dispersal, in order to better model motion of individuals beyond purely random movement. The inclusion of advection has been shown to have an impact both on the quantitative predictions of wavespeeds, and on the qualitative properties of stability and the structure of fronts (Kiselev and Ryzhik, 2001; Berestycki, 2002; Satnoianu, 2003; Berestycki and Hamel, 2007), in addition to being a framework within which to model various motion along gradients ("taxis") and

A. L. Krause

Mathematical Institute, University of Oxford, Andrew Wiles Building, Radcliffe Observatory Quarter, Woodstock Road, Oxford OX2 6GG United Kingdom

R. A. Van Gorder

Department of Mathematics and Statistics, University of Otago, P.O. Box 56, Dunedin 9054, New Zealand

E-mail: rvangorder@maths.otago.ac.nz 
other relevant dispersal mechanisms Kareiva and Odell (1987); Pettet et al (2000); Wang (2013); Hillen and Painter (2009). Traveling wave solutions have also been studied in cross-diffusion systems both theoretically (Wu, 1995; del Castillo-Negrete et al, 2002; Gambino et al, 2012; Horstmann, 2007), and in terms of modeling ecological scenarios, such as plant dispersal (Kuznetsov et al, 1994). In each of these cases, the general approaches have built off of those developed for studying traveling wave solutions in reaction-diffusion equations, and comparisons between them have given important insights on what impacts the non-diffusive dispersal mechanisms have had on the structure of traveling wave solutions.

In (Taylor et al, 2020), we studied the dispersal and clustering of populations of multiple species interacting via an extension of the model proposed by Grindrod (1988) controlling the dispersal of individuals in single and multispecies communities (Grindrod, 1988, 1991). The work of Grindrod (1988) built on simpler models by Gurtin and MacCamy (1977) and Bertsch et al (1985), by modelling behaviors where individuals can respond to threats of predation and starvation, and responses to the environment occur on a much shorter timescale than births and deaths of individuals. This motion was modelled separately from random diffusion through an advection term, with each species moving along a fecundity gradient. The dynamics of such models in one spatial domain were considered in Grindrod (1988), and in two spatial dimensions in Kurowski et al (2017). The evolution of conditional dispersal strategies in the context of competition between two species that are ecologically identical except in their dispersal mechanisms was considered in Chen et al (2008), and the spatial dependence of such dynamics was then considered in Hambrock and Lou (2009), when a spatially heterogeneous domain was considered. Much of the work on this model has been in terms of studying its pattern-forming potential, and hence as a framework to understand colony formation in population dynamics.

Previously, Ibrahim and Nasreddine (2017) proved the existence of planar waves in the one-species version of the model of Grindrod (1988) on $\mathbb{R}$, although no solutions were constructed or studied qualitatively. As far as we are aware, there have not been many studies concerned with traveling wave solutions in this kind of model. Motivated by Ibrahim and Nasreddine (2017), we study wave solutions, both planar waves and radial waves for one and two species, showing that the speed of the wavefront tends to increase with the rate of directed motion along resource gradients. We will first rely on estimates of the wavespeed from a classical linear analysis in the traveling-wave phase space. Such a linearization will turn out to predict a minimal wavespeed independent of the directed motion of the populations, in both the single and two-species cases, and hence will be equivalent to predictions from systems without this additional feature. We will then pursue an alternative approach to quantifying the impact of this advection by perturbing exact analytical expressions of traveling waves with special wave speeds, in order to capture the influence of directed motion in these models. We finally will investigate such traveling waves numerically.

Linear determinacy, when a wavefront's spreading speed (Aronson and Weinberger, 1978) is precisely predicted predicted by the linearization at the front, is an important question for reaction-diffusion systems and related models, as it indicates when simple estimates of the wavespeed can be relied upon. Substantial work has been done trying to resolve when estimates from linear stability analysis exactly match the asymptotic spreading speed, especially in the context of cooperative or competitive LotkaVolterra equations (Lewis et al, 2002; Li et al, 2005; Huang and Han, 2011; Girardin and Nadin, 2015; Girardin, 2018; Alhasanat and Ou, 2019; Girardin and Lam, 2019). Hosono (1998) first showed that such simple estimates can fail for some parameters even in the two-species Lotka-Volterra system with only diffusion, motivating much of the subsequent work. See also the review by Castillo-Chavez et al (2013) for a broader perspective. It is also known that in many cases, advective or convective terms do not enter into the analysis for pushed fronts, and hence such systems rarely have this property of linear determinacy (Al-Kiffai and Crooks, 2016). Our perturbation analysis and numerical evidence suggests that the model presented in (Taylor et al, 2020) will never satisfy this condition of linear determinacy, and we raise the question of computing the exact dependence of the asymptotic spreading speed as future work.

We first briefly review the model presented in (Taylor et al, 2020) in Section 2. We begin with the onespecies model, constructing the planar waves predicted in the work of Ibrahim and Nasreddine (2017) in Section 3. Interestingly, our analytical results extend the exact wave solution of Fisher's equation derived in Ablowitz and Zeppetella (1979) to include small magnitude advection. We show, through both analytical work and numerical simulations, that advection toward resource gradients can increase the wavespeed relative to what is found in purely reaction-diffusion systems, resulting in more rapid wavefront motion for each species, and hence in more effective motion toward underexploited resources. We then study waves involving two species, with one following the other in Section 4. Finally, we link some of the study of traveling waves to the results on pattern-formation derived in (Taylor et al, 2020) 
by showing preliminary numerical explorations of wave-initiated patterning in Section 5 . In addition to planar waves, we also numerically study radial waves, which model a radially expanding colony. Finally we conclude by discussing our results, and suggesting a variety of extensions in Section 6 .

\section{Competition model with advection toward resource gradients}

We recall the dimensionless model from Taylor et al (2020):

$$
\begin{gathered}
\frac{\partial u_{\ell}}{\partial t}=\delta_{\ell} \nabla^{2} u_{\ell}-\mu_{\ell} \nabla \cdot\left(u_{\ell} \mathbf{w}_{\ell}\right)+u_{\ell} E_{\ell}, \\
-\epsilon_{\ell} \nabla^{2} \mathbf{w}_{\ell}+\mathbf{w}_{\ell}=\nabla F_{\ell} \\
E_{\ell}\left(u_{1}, u_{2}, \ldots, u_{n}\right)=A_{\ell}-\sum_{j=1}^{n} a_{\ell j} u_{j}, \\
F_{\ell}\left(u_{1}, u_{2}, \ldots, u_{n}\right)=-\sum_{j=1}^{n} b_{\ell j} u_{j},
\end{gathered}
$$

for $\ell=1, \ldots, n$. Dimensionless boundary and initial conditions are

$$
\begin{gathered}
\mathbf{n} \cdot \mathbf{w}_{\ell}=\mathbf{n} \cdot \nabla u_{\ell}=0, \\
\mathbf{u}(\mathbf{x}, 0)=\mathbf{u}_{0}(\mathbf{x}) \geq 0 .
\end{gathered}
$$

We will assume $a_{i i}>0$ for all $i$ so that each species undergoes intraspecific competition, although the off-diagonal components can take either sign, depending on the dynamics being modelled. One can scale the density of each species to reduce some of the parameters in the kinetic functions, but we will not do so for generality and simplicity. We recall from Taylor et al (2020) that this model can be directly related to a non-local cross-diffusion formulation in a few different ways.

2.1 Cross-diffusion model in the $\epsilon_{\ell} \rightarrow 0$ limit

In the $\epsilon_{\ell}=0$ limit, we can write $\mathbf{w}_{\ell}=\nabla F_{\ell}\left(u_{1}, \ldots, u_{n}\right)$. Placing this back into the reaction-diffusion equations, we obtain

$$
\frac{\partial u_{\ell}}{\partial t}=\nabla \cdot\left(\delta_{\ell} \nabla u_{\ell}+\mu_{\ell} \sum_{j=1}^{n} b_{\ell j} u_{\ell} \nabla u_{j}\right)+u_{\ell}\left(A_{\ell}-\sum_{j=1}^{n} a_{\ell j} u_{j}\right)
$$

which is a local cross-diffusion system.

2.2 Non-local cross-diffusion model in the small- $\epsilon_{\ell}$ limit

Provided that $\epsilon_{\ell}$ is sufficiently small, note that (1b) can be solved if one obtains the Green's function for $-\varepsilon_{\ell} \nabla^{2} \mathbf{w}_{\ell}+\mathbf{w}_{\ell}$, resulting in a non-local formulation. Alternatively, one may consider a formal expansion in small $\varepsilon_{\ell}$, of the form

$$
\mathbf{w}_{\ell}=\mathbf{w}_{\ell, 0}+\varepsilon_{\ell} \mathbf{w}_{\ell, 1}+\varepsilon_{\ell}^{2} \mathbf{w}_{\ell, 2}+\cdots
$$

At lowest order, note that from this expansion we recover the $\varepsilon_{\ell}=0$ limit, $\mathbf{w}_{\ell, 0}=\nabla F_{\ell}$. Placing the expansion (3) back into (1b), and matching powers of $\varepsilon_{\ell}$, we arrive at the formal expansion

$$
\mathbf{w}_{\ell}=\sum_{q=0}^{\infty} \varepsilon_{\ell}^{q} \nabla^{2 q} \nabla F_{\ell}\left(u_{1}, \ldots, u_{n}\right)=\nabla \sum_{q=0}^{\infty} \varepsilon_{\ell}^{q} \nabla^{2 q} F_{\ell}\left(u_{1}, \ldots, u_{n}\right),
$$

which we suppose will converge for small enough $\varepsilon_{\ell}$. Placing (4) back into the reaction-diffusion equations (1a), we obtain

$$
\frac{\partial u_{\ell}}{\partial t}=\nabla \cdot\left(\delta_{\ell} \nabla u_{\ell}+\mu_{\ell} \sum_{q=0}^{\infty} \varepsilon_{\ell}^{q} u_{\ell} \nabla^{2 q+1} F_{\ell}\left(u_{1}, \ldots, u_{n}\right)\right)+u_{\ell} E_{\ell}\left(u_{1}, \ldots, u_{n}\right)
$$


which is a non-local cross-diffusion system.

As (5) involves an infinite series of differential operators, there are natural questions of convergence for this formulation. As we are concerned with travelling wave solutions, which commonly vary locally in an exponential manner, it makes sense to consider $u_{\ell}$ satisfying $\left\|\nabla^{r} u_{\ell}\right\|<C_{\ell} M_{\ell}^{r}$ for each $\ell=1,2, \ldots, n$, where $M_{\ell}>0$ is the exponential rate of growth or decay and $C_{\ell}>0$ is an $\mathcal{O}(1)$ constant in $M_{\ell}$. Then,

$$
\begin{aligned}
\left\|\nabla^{r} F_{\ell}\left(u_{1}, \ldots, u_{n}\right)\right\| & =\left\|\nabla^{r}\left(-b_{\ell 1} u_{1}-\cdots b_{\ell n} u_{n}\right)\right\| \\
& \leq\left|b_{\ell 1}\right| C_{1} M_{1}^{r}+\cdots+\left|b_{\ell n}\right| C_{n} M_{n}^{r} \\
& \leq\left(\sum_{k=1}^{n}\left|b_{\ell k}\right| C_{k}\right)\left(\max _{k=1,2, \ldots, n} M_{k}\right)^{r} .
\end{aligned}
$$

The functions $u_{\ell}$ will always be $\mathcal{O}(1)$ in $M_{\ell}$ (they are bounded by the form of the reaction kinetics $u_{\ell} E_{\ell}$ ), and so for convergence of the series in (5), we should require

$$
\epsilon_{\ell}^{q}\left(\sum_{k=1}^{n}\left|b_{\ell k}\right|\right)\left(\max _{k=1,2, \ldots, n} M_{k}\right)^{2 q+1}<1 \quad \text { as } \quad q \rightarrow \infty .
$$

Noting that the $\mathcal{O}(1)$ terms will not matter in the limit $q \rightarrow \infty$, we obtain geometric convergence of the series in (5) through the $q$ th root test provided that each $\epsilon_{\ell}$ satisfies the bound

$$
0 \leq \epsilon_{\ell}<\left(\max _{k=1,2, \ldots, n} M_{k}\right)^{-2} .
$$

We remark that non-local cross-diffusion models have been of increasing interest in the literature far a variety of biological and ecological applications (Kuznetsov et al, 1994; Hillen and Painter, 2009). One benefit of our approach is that for (1) the non-locality associated to this cross-diffusion mechanism is encoded into the $\mathbf{w}_{\ell}$ functions, and although the dimension of the state space is higher, one solves (1) as a local model. We also view (1) as easier to interpret as each term has a clear ecological significance.

\section{Planar traveling waves of a single species}

We study planar traveling waves in one-species forms of the model (1), obtaining both qualitative analytical results and numerical simulations.

\subsection{The single-species traveling wave problem}

We first consider the case of plane waves for a single species, with $\mathbf{x} \in \mathbb{R}^{n}$. For simplicity, we drop indices, and write the model as

$$
\begin{gathered}
\frac{\partial u}{\partial t}=\delta \nabla^{2} u-\mu \nabla \cdot(u \mathbf{w})+u(A-a u), \\
-\epsilon \nabla^{2} \mathbf{w}+\mathbf{w}=-b \nabla u .
\end{gathered}
$$

Making the change of variables $u(\mathbf{x}, t)=U(z), \mathbf{w}(\mathbf{x}, t)=\mathbf{W}(z)$, where the wave variable is $z=\mathbf{k} \cdot \mathbf{x}-c t$ with $c$ the wave speed, (9)-(10) becomes

$$
\begin{gathered}
\delta|\mathbf{k}|^{2} \frac{\mathrm{d}^{2} U}{\mathrm{~d} z^{2}}-\mu \mathbf{k} \cdot \frac{\mathrm{d}}{\mathrm{d} z}(U \mathbf{W})+c \frac{\mathrm{d} U}{\mathrm{~d} z}+U(A-a U)=0, \\
-\epsilon|\mathbf{k}|^{2} \frac{\mathrm{d}^{2} \mathbf{W}}{\mathrm{d} z^{2}}+\mathbf{W}=-b \mathbf{k} \frac{\mathrm{d} U}{\mathrm{~d} z} .
\end{gathered}
$$

In the case where $\epsilon=0$, note that $\mathbf{W}=-b \mathbf{k} \frac{\mathrm{d} U}{\mathrm{~d} z}$, and we have the single equation

$$
\delta|\mathbf{k}|^{2} \frac{\mathrm{d}^{2} U}{\mathrm{~d} z^{2}}+b \mu|\mathbf{k}|^{2} \frac{\mathrm{d}}{\mathrm{d} z}\left(U \frac{\mathrm{d} U}{\mathrm{~d} z}\right)+c \frac{\mathrm{d} U}{\mathrm{~d} z}+U(A-a U)=0 .
$$

In the case of $0<\epsilon \ll 1$, using (5) we have the non-local formulation

$$
\delta|\mathbf{k}|^{2} \frac{\mathrm{d}^{2} U}{\mathrm{~d} z^{2}}+b \mu|\mathbf{k}|^{2} \frac{\mathrm{d}}{\mathrm{d} z}\left(U \sum_{j=0}^{\infty} \epsilon^{j}|\mathbf{k}|^{2 j} \frac{\mathrm{d}^{2 j+1} U}{\mathrm{~d} z^{2 j+1}}\right)+c \frac{\mathrm{d} U}{\mathrm{~d} z}+U(A-a U)=0
$$

for the traveling wave. 


\subsection{Asymptotic scalings and bound on wavespeed}

Note that there are two fixed points in either of the two cases, and these are the algebraic solution of $U^{*}\left(A-a U^{*}\right)=0$, namely $U^{*}=0$ and $U^{*}=A / a$.

\subsubsection{The case of $\epsilon=0$}

First we start with $\epsilon=0$. If we consider a small perturbation about $U^{*}$, say $U=U^{*}+\sigma \tilde{U}$ where $|\sigma| \ll 1$, then for $U^{*}=0,(13)$ is linearized like

$$
\delta|\mathbf{k}|^{2} \frac{\mathrm{d}^{2} \tilde{U}}{\mathrm{~d} z^{2}}+c \frac{\mathrm{d} \tilde{U}}{\mathrm{~d} z}+A \tilde{U}=0
$$

We find that

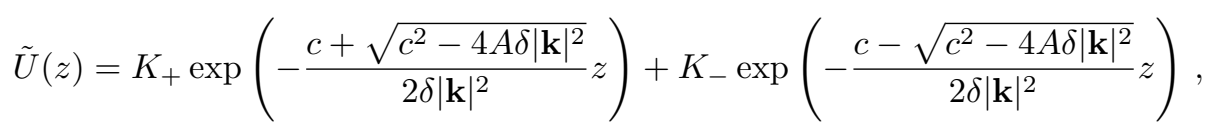

where $K_{ \pm}$are constants. Note that $U^{*}=0$ is attracting for $z \rightarrow+\infty(\tilde{U} \rightarrow 0$ as $z \rightarrow+\infty)$ and repulsive for $z \rightarrow-\infty(\tilde{U} \rightarrow \infty$ as $z \rightarrow-\infty)$, provided that $c>2 \sqrt{A \delta}|\mathbf{k}|$. We do not permit complex heteroclinic solutions, as they will oscillate into either of the steady states they connect. Therefore, we require $c>2 \sqrt{A \delta}|\mathbf{k}|$. Meanwhile, if we linearize $U$ about $U^{*}=A / a$, we find that (13) is linearized like

$$
\delta|\mathbf{k}|^{2} \frac{\mathrm{d}^{2} \tilde{U}}{\mathrm{~d} z^{2}}+\frac{b A \mu}{a}|\mathbf{k}|^{2} \frac{\mathrm{d}^{2} \tilde{U}}{\mathrm{~d} z^{2}}+c \frac{\mathrm{d} \tilde{U}}{\mathrm{~d} z}-A \tilde{U}=0 .
$$

We find that

$$
\begin{aligned}
\tilde{U}(z)= & K_{+} \exp \left(-\frac{c a+\sqrt{(c a)^{2}+4 A a(a \delta+A b \mu)|\mathbf{k}|^{2}}}{(a \delta+A b \mu)|\mathbf{k}|^{2}} z\right) \\
& +K_{-} \exp \left(-\frac{c a-\sqrt{(c a)^{2}+4 A a(a \delta+A b \mu)|\mathbf{k}|^{2}}}{(a \delta+A b \mu)|\mathbf{k}|^{2}} z\right),
\end{aligned}
$$

where $K_{ \pm}$are constants. Provided that $a \delta+A b \mu>0$, the state $U^{*}=A / a$ is repulsive for $z \rightarrow \pm \infty$ $(\tilde{U} \rightarrow \infty$ as $z \rightarrow \pm \infty)$. This suggests the existence of a heteroclinic connection $U(z)$ between $U^{*}=0$ and $U^{*}=A / a$, with $U \rightarrow A / a$ as $z \rightarrow-\infty$ and $U \rightarrow 0$ as $z \rightarrow+\infty$.

This is of course not a formal proof. Proofs of related results were found in (Dunbar, 1983, 1984), and a formal proof of the existence of such a heteroclinic connection would be quite involved in its own right, and we choose to instead provide evidence for the existence of these heteroclinic connections numerically. This heteroclinic connection corresponds to a traveling wave solution $u(\mathbf{x}, t)=U(\mathbf{k} \cdot \mathbf{x}-c t)$ with the wavefront moving from high concentration $u=A / a$ to low concentration $u=0$. Ecologically, this means that this single species moves from high concentration regions into regions of low concentration which it then colonizes. For such a solution to exist, we require $c>2 \sqrt{A \delta}|\mathbf{k}|$, and such a bound on the wavespeed is similar to other results in the literature for reaction-diffusion models, specifically the original model including logistic growth and diffusion by Fisher (1937). In particular, the advective term does not change the minimal wavespeed, though we anticipate it can influence the stability of different wavespeeds.

\subsubsection{The case of $0<\epsilon \ll 1$ under the infinite order formulation (14)}

Consider next the case of $0<\epsilon \ll 1$. Linearization about $U^{*}=0$ is identical to what was found for the $\epsilon=0$ case in (15), with the asymptotic structure given by (16), and the argument for $c>2 \sqrt{A \delta}|\mathbf{k}|$ remaining. Linearizing about $U^{*}=A / a$, we have that $\tilde{U}(z)$ obeys

$$
\delta|\mathbf{k}|^{2} \frac{\mathrm{d}^{2} \tilde{U}}{\mathrm{~d} z^{2}}+\frac{b A \mu}{a}|\mathbf{k}|^{2} \sum_{j=0}^{\infty} \epsilon^{j}|\mathbf{k}|^{2 j} \frac{\mathrm{d}^{2 j+2} \tilde{U}}{\mathrm{~d} z^{2 j+2}}+c \frac{\mathrm{d} \tilde{U}}{\mathrm{~d} z}-A \tilde{U}=0 .
$$

As this equation is linear, we assume $U(z)=\exp (\eta z)$, to obtain

$$
\delta|\mathbf{k}|^{2} \eta^{2}+\frac{b A \mu}{a} \frac{|\mathbf{k}|^{2} \eta^{2}}{1-\epsilon|\mathbf{k}|^{2} \eta^{2}}+c \eta-A=0,
$$


where we have used that

$$
\sum_{j=0}^{\infty} \epsilon^{j}|\mathbf{k}|^{2 j} \eta^{2 k+2}=\frac{\eta^{2}}{1-\epsilon|\mathbf{k}|^{2} \eta^{2}}
$$

which is valid for $|\eta|<\frac{1}{\sqrt{\epsilon}|\mathbf{k}|}$. Since $A>0$, observe that (20) has two real roots $\eta^{*}$ and $\eta^{* *}$ satisfying

$$
-\frac{1}{\sqrt{\epsilon}|\mathbf{k}|}<\eta^{* *}<0<\eta^{*}<\frac{1}{\sqrt{\varepsilon}|\mathbf{k}|}
$$

provided that $a \delta+A b \mu>0$. Since these roots always fall in the interval bounded by $|\eta|<\frac{1}{\sqrt{\epsilon}|\mathbf{k}|}$, the operation (21) is well-defined and hence there exists one root which is always positive and one root which is always negative. As such, $U^{*}=A / a$ is repulsive for both $z \rightarrow \pm \infty$, which is the same as what was found in the $\epsilon=0$ case. Similar to the $\epsilon=0$ case, this suggests the existence of a heteroclinic connection $U(z)$ between $U^{*}=0$ and $U^{*}=A / a$, with $U \rightarrow A / a$ as $z \rightarrow-\infty$ and $U \rightarrow 0$ as $z \rightarrow+\infty$, with the minimal wavespeed the same as for the $\epsilon=0$ case discussed above.

We remark that the bound $|\eta|<\frac{1}{\sqrt{\epsilon}|\mathbf{k}|}$ is actually a restatement of (8). Indeed, we can rearrange this inequality to find

$$
\epsilon<(\eta|\mathbf{k}|)^{-2}
$$

and hence we can see this is a bound for how small $\epsilon$ needs to be in order to permit a travelling wave solution to (5). This makes sense, as the linearized problem requires a solution which scales like $\exp (\eta(\mathbf{k} \cdot \mathbf{x})-c t)$, for which $\left\|\nabla^{r} u\right\|<(\eta|\mathbf{k}|)^{r}$. Identifying $M=\eta|\mathbf{k}|>0$ (the exponential rate of growth or decay) and $C=1$, then (8) reduces exactly to (23).

\subsubsection{The case of $0<\epsilon \ll 1$ under the coupled system (11)-(12)}

While the infinite order formulation is convenient, as it results in a scalar equation for the waveform $u$, it is natural to worry that such a formulation may fail to converge or otherwise miss something from the full system (11)-(12). We therefore consider the asymptotic scalings from the full system (11)-(12), directly.

If we consider a small perturbation about $U^{*}$, taking $U=U^{*}+\sigma \tilde{U}$ and $\mathbf{W}=\sigma \tilde{\mathbf{W}}$, where $|\sigma| \ll 1$. For $U^{*}=0,(11)-(12)$ is linearized like

$$
\begin{gathered}
\delta|\mathbf{k}|^{2} \frac{\mathrm{d}^{2} \tilde{U}}{\mathrm{~d} z^{2}}+c \frac{\mathrm{d} \tilde{U}}{\mathrm{~d} z}+A \tilde{U}=0, \\
-\epsilon|\mathbf{k}|^{2} \frac{\mathrm{d}^{2} \tilde{\mathbf{W}}}{\mathrm{d} z^{2}}+\tilde{\mathbf{W}}=-b \mathbf{k} \frac{\mathrm{d} \tilde{U}}{\mathrm{~d} z} .
\end{gathered}
$$

Assuming $\tilde{U}=\exp (\eta z)$ and $\tilde{\mathbf{W}}=w_{0} \exp (\eta z) \mathbf{k}$, these differential equations reduce to the algebraic equations

$$
\begin{gathered}
\delta|\mathbf{K}|^{2}+c \eta+A=0, \\
\left(-\epsilon \eta^{2}|\mathbf{k}|^{2}+1\right) w_{0}=-b \eta .
\end{gathered}
$$

From (26), we see that $U$ is completely independent of $\mathbf{W}$ at $\mathcal{O}(\sigma)$, and the behaviour near $U^{*}=0$ is exactly the same as previously described by (16). After similar reasoning, we again require $c>2 \sqrt{A \delta}|\mathbf{k}|$.

Next linearizing about $U^{*}=A / a$, we obtain the linear system

$$
\begin{gathered}
\delta|\mathbf{k}|^{2} \frac{\mathrm{d}^{2} \tilde{U}}{\mathrm{~d} z^{2}}-\mu \frac{A}{a} \mathbf{k} \cdot \frac{d \tilde{\mathbf{W}}}{d z}+c \frac{\mathrm{d} \tilde{U}}{\mathrm{~d} z}-A \tilde{U}=0, \\
-\epsilon|\mathbf{k}|^{2} \frac{\mathrm{d}^{2} \tilde{\mathbf{W}}}{\mathrm{d} z^{2}}+\tilde{\mathbf{W}}=-b \mathbf{k} \frac{\mathrm{d} \tilde{U}}{\mathrm{~d} z} .
\end{gathered}
$$

As this system is linear, we assume $U(z)=\exp (\eta z)$ and $\tilde{\mathbf{W}}=w_{0} \exp (\eta z) \mathbf{k}$, obtaining the algebraic system

$$
\begin{gathered}
\delta|\mathbf{k}|^{2} \eta^{2}-\frac{A \mu}{a} w_{0} \eta|\mathbf{k}|^{2}+c \eta-A=0, \\
\left(-\epsilon \eta^{2}|\mathbf{k}|^{2}+1\right) w_{0}=-b \eta .
\end{gathered}
$$


Solving (31) for the constant $w_{0}$, we find

$$
w_{0}=-\frac{b \eta}{1-\epsilon \eta^{2}|\mathbf{k}|^{2}}
$$

and substituting $w_{0}$ into $(30)$, we obtain

$$
\delta|\mathbf{k}|^{2} \eta^{2}+\frac{b A \mu}{a} \frac{|\mathbf{k}|^{2} \eta^{2}}{1-\epsilon|\mathbf{k}|^{2} \eta^{2}}+c \eta-A=0
$$

which is exactly the same as (20). The constant $w_{0}$ only makes sense for $|\eta|<\frac{1}{\sqrt{\epsilon}|\mathbf{k}|}$, and this is the same restriction we obtained in the analysis of the infinite order equation (14). In other words, we again require $\epsilon$ to satisfy the bound (23).

Since $A>0$, observe that (33) again has two real roots $\eta^{*}$ and $\eta^{* *}$ satisfying (22), provided that $a \delta+A b \mu>0$. One root is always positive and one root which is always negative, so $U^{*}=A / a$ is repulsive for both $z \rightarrow \pm \infty$, suggesting the existence of a heteroclinic connection $U(z)$ between $U^{*}=0$ and $U^{*}=A / a$, with $U \rightarrow A / a$ as $z \rightarrow-\infty$ and $U \rightarrow 0$ as $z \rightarrow+\infty$. As such, the asymptotic scalings from the system (11)-(12) are equivalent to those found previously from the scalar but infinite order formulation (14).

\subsection{Perturbation approximation of a traveling wave solution}

One interesting result for Fisher's equation is the existence of an exact solution with a special wave speed (Ablowitz and Zeppetella, 1979). However, when $\mu \neq 0$, we do not have such a solution for the corresponding equation $(E(u)=1-u)$. Actually, we can show that there will exist no closed-form exact solutions of the kind found in Ablowitz and Zeppetella (1979). This demonstrates that the equations we consider with advection are fundamentally more difficult to deal with than Fisher's equation or systems of such equations. In particular, we can show that our equation is not conditionally integrable, which was true of Fisher's equation.

One method for analytically constructing a nonlinear wave solution to a reaction-diffusion equation is to exploit conditional integrability and construct a solution through dominant balance provided the equation passes the Painlevé test. This approach was used in the construction of an exact solution to Fisher's equation in (Ablowitz and Zeppetella, 1979), and has been applied to study a variety of other conditionally integrable reaction or reaction-diffusion equations; for more information, see (Goriely, 1996; Hearns et al, 2012; Ramani et al, 1989; Russo et al, 2013; Weiss et al, 1983; Weiss, 1983). To begin, consider the case of $\epsilon=0$, so that we have (13). We attempt to find a dominant balance for this equation, consider an expansion about the singular manifold $z=z_{0}$, as given by the terminating Laurent expansion

$$
U(z)=\frac{U_{-\alpha}}{\left(z-z_{0}\right)^{\alpha}}+\text { higher order terms }
$$

With this, the advection term in (13) dominates with order $\left(z-z_{0}\right)^{-2 \alpha-2}$ and for $\alpha>0$ there is no term to balance it. Any attempt at other balances would give $\alpha<0$, in which case there is no singular manifold. Hence when $\epsilon=0$ the traveling wave equation is not integrable for $\mu \neq 0$. A similar attempt can be made with $\epsilon>0$, and in this case, we can actually show that there exist Laurent series expansions

$$
U(z)=\frac{U_{-2}}{\left(z-z_{0}\right)^{2}}+\text { higher order terms and } \mathbf{W}(z)=\frac{W_{-1}}{\left(z-z_{0}\right)^{1}} \mathbf{k}+\text { higher order terms },
$$

so the Laurent series truncates at a finite index. However, when calculating the coefficients in the Laurent series, we find that the leading terms all must vanish when $\mu \neq 0$. As such, we cannot use the approach to construct an exact solution, as done in Ablowitz and Zeppetella (1979).

As the exact solution of Ablowitz and Zeppetella (1979) exists in the $\mu=0$ limit for the one-species model, it is natural to consider the case where $\mu \ll 1$ in order to determine how this will modify the exact solution. This would correspond to a perturbation of the exact heteroclinic connection which exists for the $\mu=0$ case. To simplify our analysis, we make the change of variable

$$
y=\exp (\beta z), \quad U(z)=V(y), \quad \mathbf{W}(z)=-Q(y) \mathbf{k},
$$


where $\beta>0$ is a parameter to be determined. Equation (36) puts (11)-(12) into the form

$$
\begin{gathered}
\delta|\mathbf{k}|^{2} \beta^{2}\left(y \frac{\mathrm{d} V}{\mathrm{~d} y}+y^{2} \frac{\mathrm{d}^{2} V}{\mathrm{~d} y^{2}}\right)+\mu|\mathbf{k}|^{2} \beta y \frac{\mathrm{d}}{\mathrm{d} y}(Q V)+c \beta y \frac{\mathrm{d} V}{\mathrm{~d} y}+V(A-a V)=0, \\
\varepsilon|\mathbf{k}|^{2} \beta^{2}\left(y \frac{\mathrm{d} Q}{\mathrm{~d} y}+y^{2} \frac{\mathrm{d}^{2} Q}{\mathrm{~d} y^{2}}\right)-Q+b \beta y \frac{\mathrm{d} V}{\mathrm{~d} y}=0 .
\end{gathered}
$$

When $\mu=0$, the equations decouple and one can solve for $V$, obtaining the exact solution

$$
V(y)=\frac{A / a}{(1+\zeta y)^{2}}
$$

where $\zeta$ is an arbitrary positive constant. This solution exists provided that $4 \beta^{2} \delta|\mathbf{k}|^{2}-2 \beta c+A=0$ and $2 \beta^{2} \delta|\mathbf{k}|^{2}+2 \beta c-2 A=0$, and these conditions give

$$
c=5 \sqrt{\frac{A \delta}{6}}|\mathbf{k}| \text { and } \beta=\sqrt{\frac{A}{6 \delta}} \frac{1}{|\mathbf{k}|} .
$$

Transforming (39) back into original coordinates, we obtain the exact solution

$$
\begin{aligned}
u(\mathbf{x}, t) & =U(z)=\frac{A}{a}\left\{1+\zeta \exp \left(\sqrt{\frac{A}{6 \delta}} \frac{z}{|\mathbf{k}|}\right)\right\}^{-2} \\
& =\frac{A}{a}\left\{1+\zeta \exp \left[\sqrt{\frac{A}{6 \delta}}\left(\frac{\mathbf{k} \cdot \mathbf{x}-5 \sqrt{\frac{A \delta}{6}}|\mathbf{k}| t}{|\mathbf{k}|}\right)\right]\right\}^{-2},
\end{aligned}
$$

which is the exact solution of (37) when $\mu=0$, and corresponds to the exact solution of Ablowitz and Zeppetella (1979) in our notation. The constant $\zeta$ may be calibrated so that the solution will match certain specified initial data.

While no exact solution appears to exist for $\mu \neq 0$, we can construct a perturbation solution to determine the qualitative influence of advection. We assume

$$
V(y)=V_{0}(y)+\mu V_{1}(y)+O\left(\mu^{2}\right) \text { and } \quad Q(y)=Q_{0}(y)+O(\mu),
$$

and shall also assume that the wave speed can be represented by $c=c_{0}+\mu c_{1}+O\left(\mu^{2}\right)$. We shall neglect $\epsilon$, setting $\epsilon=0$, as we expect the qualitative behaviour to be similar between the $\epsilon=0$ and $\epsilon>0$ cases, yet neglecting $\epsilon$ permits a local formulation which is solvable at the first-order perturbation. We later show the effect of $\epsilon$ when providing numerical simulations.

Placing these expansions into (37)-(38), we note that $V_{0}(y)$ satisfies (37) with $\mu=0$, and hence its solution is (39), while $c_{0}=5 \sqrt{\frac{A \delta}{6}}|\mathbf{k}|$. Meanwhile, at next order, $V_{1}(y)$ and $Q_{0}(y)$ satisfy

$$
\begin{gathered}
\delta|\mathbf{k}|^{2} \beta^{2}\left(y \frac{\mathrm{d} V_{1}}{\mathrm{~d} y}+y^{2} \frac{\mathrm{d}^{2} V_{1}}{\mathrm{~d} y^{2}}\right)+\mu|\mathbf{k}|^{2} \beta y \frac{\mathrm{d}}{\mathrm{d} y}\left(Q_{0} V_{1}\right)+c_{0} \beta y \frac{\mathrm{d} V_{1}}{\mathrm{~d} y}+c_{1} \beta y \frac{\mathrm{d} V_{0}}{\mathrm{~d} y}+\left(A-2 a V_{0}\right) V_{1}=0, \\
-Q_{0}+b \beta y \frac{\mathrm{d} V_{0}}{\mathrm{~d} y}=0 .
\end{gathered}
$$

We find (44) has solution

$$
Q_{0}(y)=-\frac{2 \zeta A}{a} \frac{\beta y}{(1+\zeta y)^{3}},
$$

and placing this into (43), we obtain an ODE for $V_{1}(y)$ alone. Solving the resulting second-order ODE, we obtain two homogeneous solutions along with a particular solution. As $V_{0}(y)$ already holds the boundary data, we remove the two homogeneous solutions. To ensure regularity and boundedness of the particular solution, we will determine permissible values of $c_{1}$. In the limit $y \rightarrow \infty$, the particular solution tends to zero, as required. However, in the limit $y \rightarrow 0^{+}$, the particular solution diverges for generic parameter values. However, the term which diverges multiplies a parameter combination, and upon setting

$$
45 A^{2} b \sqrt{\delta}|\mathbf{k}|-812 \sqrt{6 A} a \delta c_{1}=0,
$$




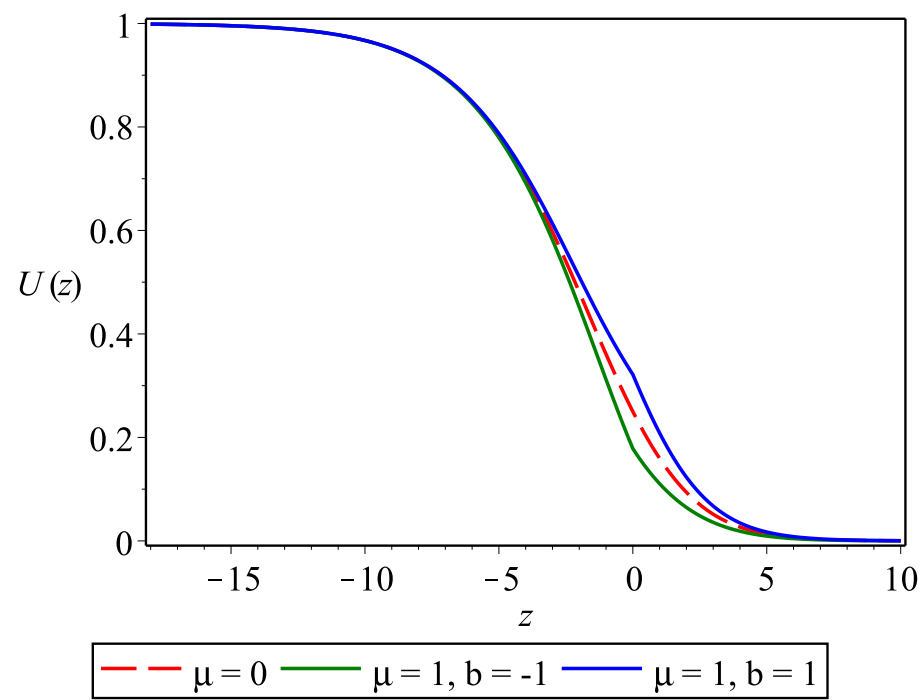

Fig. 1 Perturbation solution (49) for various values of $\mu$ and $b$. We fix $A=a=\delta=|\mathbf{k}|=1$ and choose the arbitrary constant $\zeta=1$. The solution for $\mu=0$ corresponds to the exact solution to Fisher's equation. When $b>0$, the wavefront is larger than the Fisher wavefront, whereas when $b<0$ the wavefront is smaller than the Fisher wavefront. Additionally, the wave propagates faster (slower) for $b>0(b<0)$. We plot this solution over the wave variable $z$, and note that the wavefront will move to the right in original coordinates $(\mathbf{x}, t)$.

this secular divergence is removed. From this regularity condition, we find

$$
c_{1}=\frac{15 \sqrt{6}}{1624} \frac{A^{3 / 2} b|\mathbf{k}|}{a \sqrt{\delta}} .
$$

The solution $V_{1}(y)$ is then given by

$$
\begin{aligned}
V_{1}(y)= & \frac{A^{2} b}{a^{2} \delta}\left\{\frac{857 \zeta y \ln (y)}{2842(1+\zeta y)^{3}}-\frac{406(\zeta y)^{7}-135(1+\zeta y)^{7}}{1421(\zeta y)^{6}(1+\zeta y)^{3}} \ln (1+\zeta y)\right. \\
& -\frac{9}{5684} \frac{70(\zeta y)^{7}+60(\zeta y)^{6}-450(\zeta y)^{5}-1450(\zeta y)^{4}-1975(\zeta y)^{3}-1437(\zeta y)^{2}-549(\zeta y)-87}{(\zeta y)^{6}(1+\zeta y)^{3}} \\
& \left.-\frac{56(\zeta y)^{7}-756(\zeta y)^{6}-1512(\zeta y)^{5}-1890(\zeta y)^{4}-1512(\zeta y)^{3}-756(\zeta y)^{2}-216(\zeta y)-27}{56(\zeta y)^{6}(1+\zeta y)^{4}}\right\} .
\end{aligned}
$$

One may verify that $V_{1} \rightarrow 0$ as either $y \rightarrow 0^{+}$or $y \rightarrow \infty$, as required.

With this, we have constructed the first-order perturbation theory under small $\mu$ for a traveling wave solution, and this reads

$$
\begin{aligned}
u(\mathbf{x}, t) & =U(z) \\
& =\frac{A}{a}\left\{1+\zeta \exp \left(\sqrt{\frac{A}{6 \delta}} \frac{x-c t}{|\mathbf{k}|}\right)\right\}^{-2}+\mu V_{1}\left(\exp \left(\sqrt{\frac{A}{6 \delta}} \frac{x-c t}{|\mathbf{k}|}\right)\right)+\mathcal{O}\left(\mu^{2}\right),
\end{aligned}
$$

where the approximate wavespeed reads

$$
\begin{aligned}
c & =5 \sqrt{\frac{A \delta}{6}}|\mathbf{k}|+\mu \frac{15 \sqrt{6}}{1624} \frac{A^{3 / 2} b|\mathbf{k}|}{a \sqrt{\delta}}+\mathcal{O}\left(\mu^{2}\right) \\
& =5 \sqrt{\frac{A \delta}{6}}|\mathbf{k}|\left(1+\frac{9 A b}{812 a} \frac{\mu}{\delta}+\mathcal{O}\left(\mu^{2}\right)\right) .
\end{aligned}
$$

We plot the perturbation solution (49) in Figure 1 for fixed parameters, and compare it with the classical Fisher traveling wave profile. This perturbation solution suggests that $\operatorname{sgn}\left(\frac{\partial c}{\partial \mu}\right)=\operatorname{sgn}(b)$, with $c$ increasing with an increase in $\mu$ provided that the motion is toward the fecundity gradient $(b>0)$ and 
$c$ decreasing with an increase in $\mu$ provided that the motion is away from the fecundity gradient $(b<0)$, which makes intuitive sense and is consistent with findings for other models (Kiselev and Ryzhik, 2001). With this, we have determined how a Fisher-type traveling wave should change under small advection along resource gradients.

\subsection{Numerical simulations}

Here we present numerical solutions of the one-species model in one spatial dimension. We computed these by discretizing (1) using centered finite differences in the spatial derivatives and using the MATLAB function 'ODE45' which implements a Runge-Kutta scheme due to Dormand and Prince (1980). These simulations were also checked against implementations in COMSOL, and quantitatively comparable results obtained on sufficiently fine grids. To ensure accuracy in computing the wavespeeds, we set the absolute and relative tolerances to $10^{-9}$, respectively, and constrain the maximum time step to ensure accuracy of the scheme. Fixed time step simulations using the standard 4-step Runge-Kutta method were also carried out to check convergence in the time and space steps. We considered the one-dimensional domain $x \in[0,1000]$ and took a spatial step length of $\Delta x=0.25$ so that we tracked the solution at 4,000 nodal points. We show results for various values of $\mu$ and $\epsilon$. The initial data were taken to be a smoothed step-function over the first $4 \%$ of the domain, namely $u(x, 0)=(\tanh (3(4-x))+1) / 2$.

In Figure 2 we demonstrate the effect of varying $\epsilon$ and $\mu$ in solutions for a one-species wave by plotting the profiles of $u$ with different values of these parameters. We note that the wave profiles do not appear to qualitatively change, but there is a noticeable increase in the wavespeed due to the advection as $\mu$ increases, as predicted by the perturbation theory. We also note that there is a more gradual increase in the wavespeed due to increasing $\epsilon$, and suspect this is due to its nonlocal nature influencing the 'pushed front' aspect of the wave. As suggested by the analytics, wavefronts move from regions of high concentrations into region of low concentrations (left to right for this configuration), meaning that populations $u$ migrate into unpopulated zones.

In Figure 3 we plot the numerically computed wavespeeds to confirm that these trends are generic throughout the parameter space. The influence of $\epsilon$ and $\mu$ on the wavespeed $c$ depends on both parameters, in that they amplify one another in a multiplicative way. As anticipated above, while the minimal wavespeed is comparable to the Fisher wavespeed in all cases, the realized wavespeed for this initial data varies substantially with the advection strength and $\epsilon$, which controls how strongly nonlocal the advection is. 


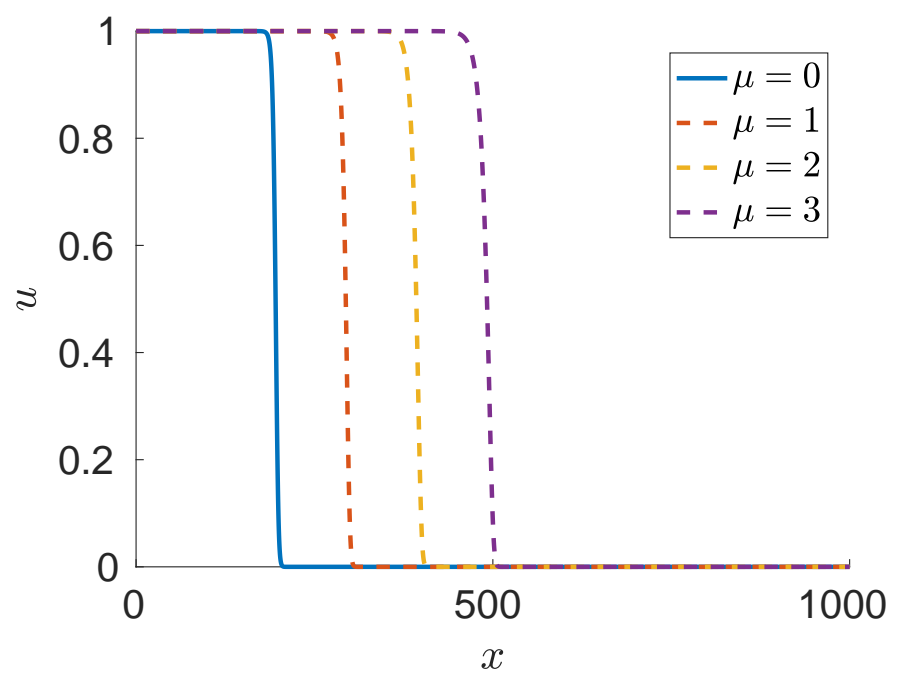

(a)

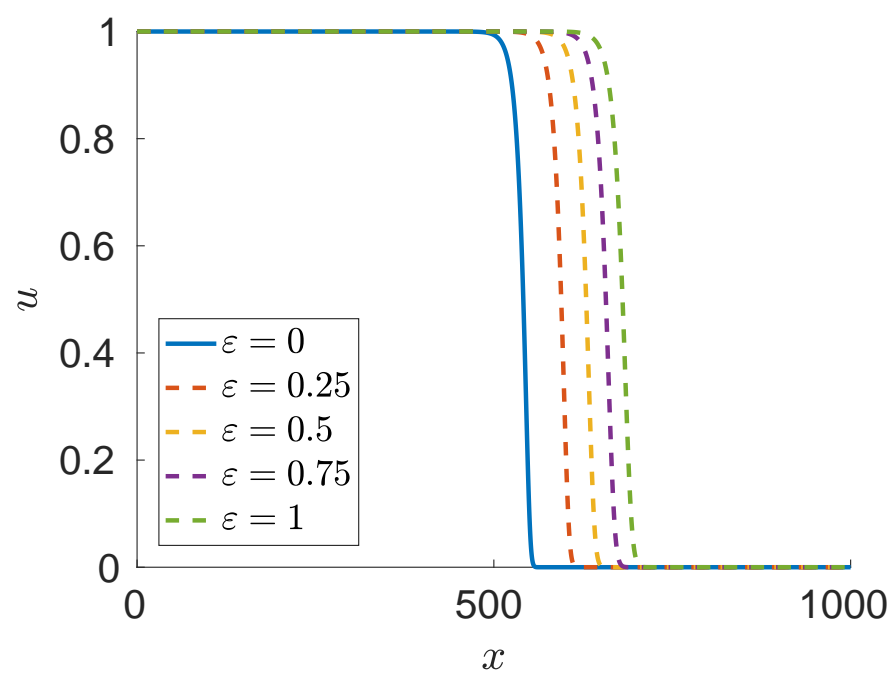

(b)

Fig. 2 Final wave profiles $u(x, t=30)$ for $A=a=b=\delta=1$ and varying values of $\mu$ and $\epsilon$. In (a) we set $\epsilon=0$, and in (b) we set $\mu=3.5$. An increase in either $\mu$ or $\epsilon$ results in an increase in the wavespeed. 


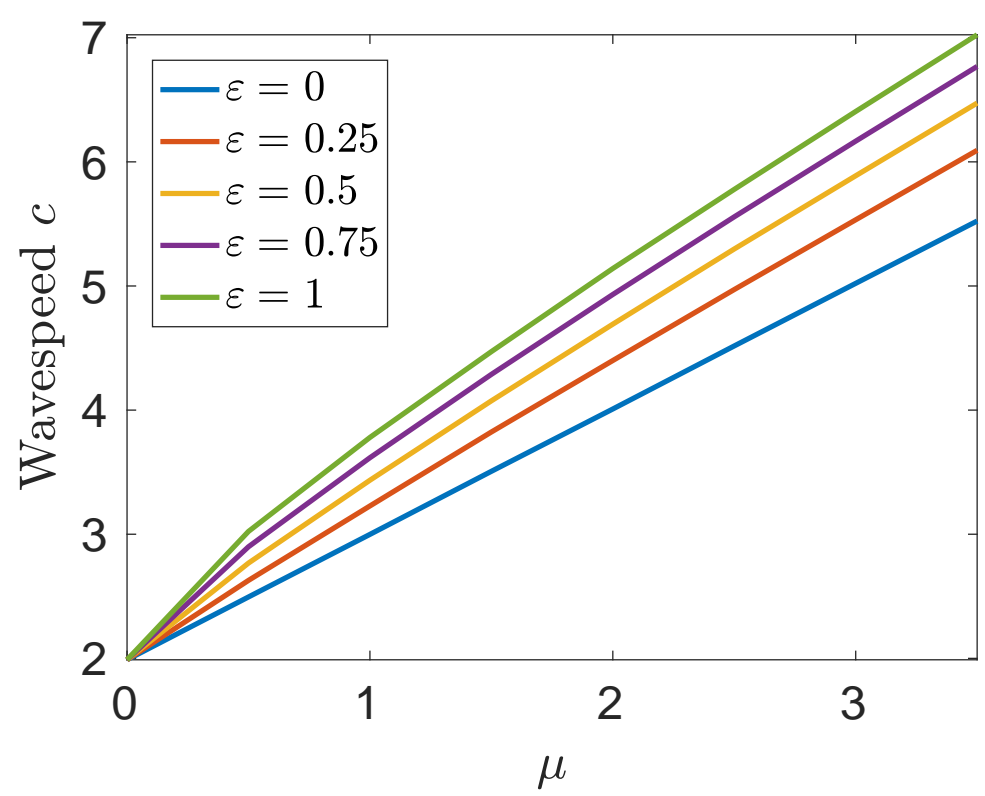

Fig. 3 Numerically computed wavespeeds for single-species waves with $A=a=b=\delta=1$, for various values of $\mu$ and $\epsilon$. As predicted, an increase in either $\mu$ or $\epsilon$ results in an increase in the wavespeed. 


\section{Two-species planar and radial traveling waves}

Motivated by existing work on reaction-diffusion models, we study planar traveling waves in two-species forms of the model (1), obtaining both qualitative analytical results and numerical simulations. We also provide evidence supporting the existence of radial waves by way of numerical simulations, and we compare these waves to their more common planar counterparts. We start with the single-species model, and later consider the two-species case.

\subsection{Exact solutions}

Making the change of variables $u_{\ell}(\mathbf{x}, t)=U_{\ell}(z), \mathbf{w}_{\ell}(\mathbf{x}, t)=\mathbf{W}_{\ell}(z)$, where the wave variable is $z=\mathbf{k} \cdot \mathbf{x}-c t$ with $c$ the wave speed, we obtain from (1) that

$$
\begin{gathered}
\delta_{\ell}|\mathbf{k}|^{2} \frac{\mathrm{d}^{2} U_{\ell}}{\mathrm{d} z^{2}}-\mu_{\ell} \mathbf{k} \frac{\mathrm{d}}{\mathrm{d} z}\left(U_{\ell} \mathbf{W}_{\ell}\right)+c \frac{\mathrm{d} U_{\ell}}{\mathrm{d} z}+U_{\ell}\left(A_{\ell}-a_{\ell 1} U_{1}-a_{\ell 2} U_{2}\right)=0, \\
-\epsilon_{\ell}|\mathbf{k}|^{2} \frac{\mathrm{d}^{2} \mathbf{W}_{\ell}}{\mathrm{d} z^{2}}+\mathbf{W}_{\ell}=-b_{\ell 1} \mathbf{k} \frac{\mathrm{d} U_{1}}{\mathrm{~d} z}-b_{\ell 2} \mathbf{k} \frac{\mathrm{d} U_{2}}{\mathrm{~d} z}
\end{gathered}
$$

where $\ell=1,2$. We have four spatially homogeneous steady states, defined by

$$
\begin{gathered}
\mathbf{S}_{0}=(0,0), \\
\mathbf{S}_{1}=\left(\frac{A_{1}}{a_{11}}, 0\right), \\
\mathbf{S}_{2}=\left(0, \frac{A_{2}}{a_{22}}\right), \\
\mathbf{S}_{3}=\left(S_{31}, S_{32}\right)=\left(\frac{A_{2} a_{12}-A_{1} a_{22}}{a_{11} a_{22}-a_{12} a_{21}}, \frac{A_{1} a_{21}-A_{2} a_{11}}{a_{11} a_{22}-a_{12} a_{21}}\right) .
\end{gathered}
$$

We shall primarily be concerned with the scenario where one species is prevalent toward one far-field, and the other species is prevalent toward the other far-field. Therefore, we will be most interested in constructing heteroclinic solutions connecting the steady states (52b) and (52c). The analytical construction of such states is beyond the scope of this paper, but we can give some qualitative analytical results. Using the approach of Section 3.2, we linearize around each of the steady states (52b) and (52c), to find that the wavespeed is bounded exactly as the purely diffusive Lotka-Volterra models are, with

$$
c^{2}>\left(c^{*}\right)^{2}=4|\mathbf{k}|^{2} \max \left\{\frac{A_{2} a_{11}-A_{1} a_{21}}{a_{11}} \delta_{2}, \frac{A_{1} a_{22}-A_{2} a_{12}}{a_{22}} \delta_{1}\right\} .
$$

In a very specific parameter regime, it is possible to find exact solutions which are generalizations of the exact solution to Fisher's equation, again for the advection-free case of $\mu=0$. We shall demonstrate this for the solution $\left(\frac{A_{1}}{a_{11}}, 0\right) \rightarrow\left(0, \frac{A_{2}}{a_{22}}\right)$. To obtain an exact solution, we will need to assume $\frac{A_{1}}{a_{11}}=\frac{A_{2}}{a_{22}}$. We furthermore assume that $a_{21}=a_{11}, a_{22}=a_{12}$, and $\delta_{2}=\delta_{1}$. Taking these parameter restrictions, and following an approach similar to that employed in Section 3.3, we find the exact solution

$$
U_{1}(z)=\frac{A_{1}}{a_{11}}\left\{1+\zeta \exp \left(\frac{1}{\sqrt{6}} \sqrt{\frac{\left(a_{11}-a_{12}\right) A_{1}}{a_{11} \delta_{1}}} \frac{z}{|\mathbf{k}|}\right)\right\}^{-2}
$$

and

$$
U_{2}(z)=\frac{A_{1}}{a_{11}}\left(1-\left\{1+\zeta \exp \left(\frac{1}{\sqrt{6}} \sqrt{\frac{\left(a_{11}-a_{12}\right) A_{1}}{a_{11} \delta_{1}}} \frac{z}{|\mathbf{k}|}\right)\right\}^{-2}\right)
$$

where the wavespeed is given by

$$
c=\frac{5}{\sqrt{6}} \sqrt{\frac{\left(a_{11}-a_{12}\right) A_{1} \delta_{1}}{a_{11}}}|\mathbf{k}|,
$$

and $\zeta>0$ is an arbitrary constant which determines the location of the wave at $t=0$. This exact solution only holds for $a_{11}>a_{12}$, requiring the intra-specific competition to dominate any inter-specific 
competition. We remark that the wavespeed (55) is slightly above the theoretical threshold predicted by (53) which reduces to $c>c^{*}$, where

$$
c^{*}=2 \sqrt{\frac{\left(a_{11}-a_{12}\right) A_{1} \delta_{1}}{a_{11}}}|\mathbf{k}|
$$

is the threshold wavespeed for the parameter regime which permits (54).

Similar to what has been discussed in Section 3.3, exact solutions do not appear to exist when $\mu \neq 0$. As we considered in Section 3.3, in the case of small advection, it is possible to seek perturbation solutions in order to correct the solution profiles and the wavespeed $c$. The approach for extending the exact wave solution (54) to the case of small yet non-zero $\mu$ is similar to that carried out in Section 3.3 for the single species wave, although much more analytically messy, and we omit the lengthy expressions. Note that since there are a number of parameters $b_{\ell j}$ involved in the advection, whether advection speeds up or slows down a wave solution will vary from case to case.

\subsection{Numerical simulations}

We now present numerical solutions of the two-species model in one spatial dimension. We computed these using the same methods as described at the beginning of Section 3.4, but considered the smaller domain $x \in[0,100]$ using a spatial step-size of $\Delta x=0.1$. The initial data were taken to be a smoothed step-function over the first $5 \%$ of the domain, namely $u_{1}(x, 0)=(\tanh (3(20-x))+1) / 2$ and $u_{2}(x, 0)=$ $1-u_{1}(x, 0)$. This corresponds to an invasive species, $u_{1}$, which is assumed to locally out-compete the more abundant species $u_{2}$. Many other simulations with different initial data and parameters were explored and demonstrated comparable behaviours for competitive dynamics.

In Figure 4 we demonstrate the effect of varying $\epsilon$ and $\mu$ in solutions of (1). Note that only $u_{1}$ is shown, but the profiles for $u_{2}$ correspond to the expected mirrored profiles, roughly of the form $u_{2} \approx 1-u_{1}$ away from the wave front. As in the single-species case, the wave profiles do not change qualitatively, but there is a noticeable increase in the wavespeed due to the advection as $\mu$ increases. In contrast to the single-species case, however, we note that increasing $\epsilon$ leads to a reduced wavespeed, likely due to non-local interactions between both species.

In Figure 5 we plot the numerically computed wavespeeds to confirm that these trends are generic throughout the parameter space. As before we see a substantial increase in the wavespeed as a function of $\mu$, but now we observe a reduction in this wavespeed due to $\epsilon$ which appears smaller than the increase due to the presence of advection, but does suggest that the non-local problem in the two-species case is markedly different from the one-species case. In particular, due to interaction between species, the wavefront is slowed considerably, and non-local interactions slow the wavefront further.

\subsection{Simulations of radially symmetric waves}

We now present numerical solutions of the two-species model in two spatial dimensions to demonstrate curvature effects, demonstrating the emergence and dynamics of radially symmetric waves (Jones, 1983; Roussier, 2004) for the model (1). We computed these using finite element solver ComsoL, and checked one spatial dimensional simulations against those computed using methods from Section 3.4, in addition to performing time and space convergence checks. We used 24,912 finite elements which gave a total of 306,174 degrees of freedom for all independent variables. We consider initial data that evolved into planar and circular wave fronts. The first of these is analogous to the one-dimensional simulations in Section 4 , namely $u_{1}(\mathbf{x}, 0)=\left(\tanh \left(3\left(20-x_{1}\right)\right)+1\right) / 2$ and $u_{2}(\mathbf{x}, 0)=1-u_{1}(\mathbf{x}, 0)$ in the domain $\mathbf{x} \in[0,100]^{2}$. The circular wave fronts were generated replacing the initial condition with $u_{1}(\mathbf{x}, 0)=(\tanh (3(20-$ $\left.\left.\left.\sqrt{x_{1}^{2}+x_{2}^{2}}\right)\right)+1\right) / 2$ in the domain $\mathbf{x} \in[-100,100]^{2}$.

In Figure 6, we plot values of $u_{1}$ over the course of these simulations over the two-dimensional domains. We note that the circular waves appear to move more slowly than the planar waves, which have speeds approximately identical to planar waves in one spatial dimension.

To confirm this curvature effect, we consider one dimensional slices from $x_{1}=0$ to $x_{1}=100$ with $x_{2}=0$ fixed in both domains, plotted in Figure 7 . We observe a definite decrease in the wave speed of the circular front, and attribute this effect to the local curvature. The influence of curvature on wavespeed has been well-studied in traveling waves in excitable media (Kenner, 1986, 1991), though we do not pursue the derivation of an Eikonal equation for the wavespeed here. 


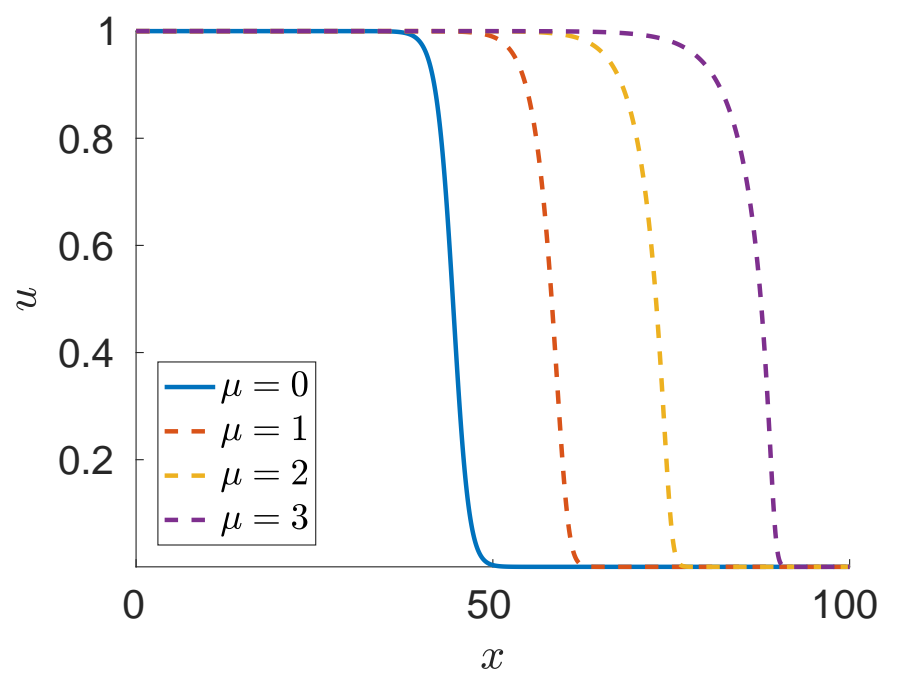

(a)

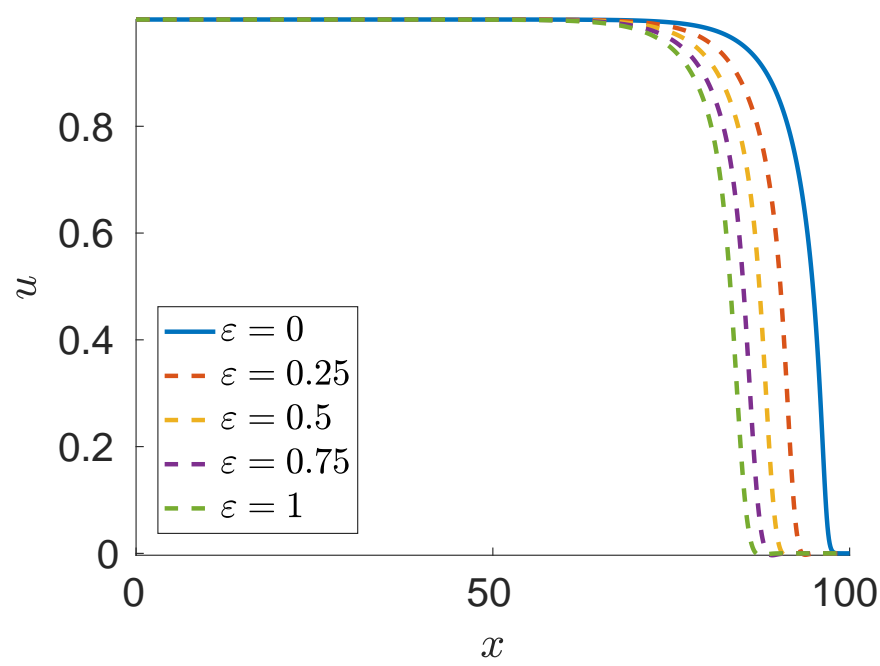

(b)

Fig. 4 Planar wave profiles $u_{1}(x, 100)$ for $\delta_{\ell}=A_{\ell}=1$ with $a_{11}=a_{22}=1, a_{12}=2, a_{21}=3, b_{\ell j}=a_{\ell j}$, and with varying values of $\mu_{i} \equiv \mu$ and $\epsilon_{i} \equiv \epsilon$. In (a) we fix $\epsilon=0$, and in (b) we fix $\mu=3.5$. 


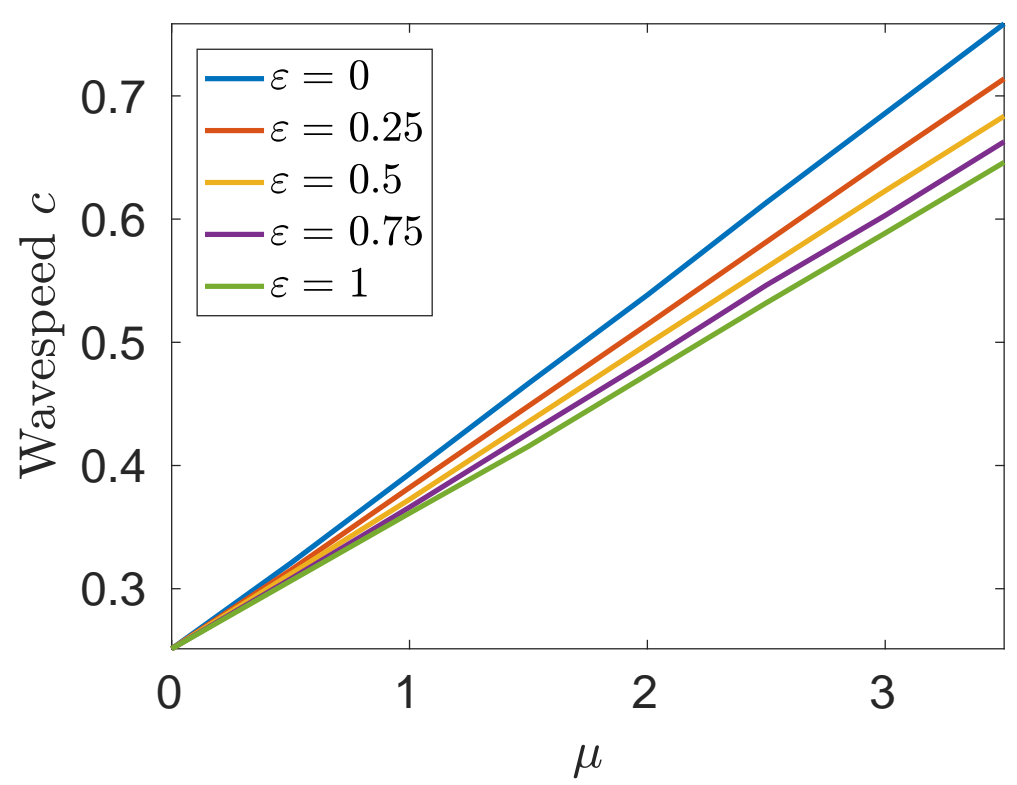

Fig. 5 Wavespeeds $c$ for two-species waves computed numerically using the same parameter values as in Figure 4.
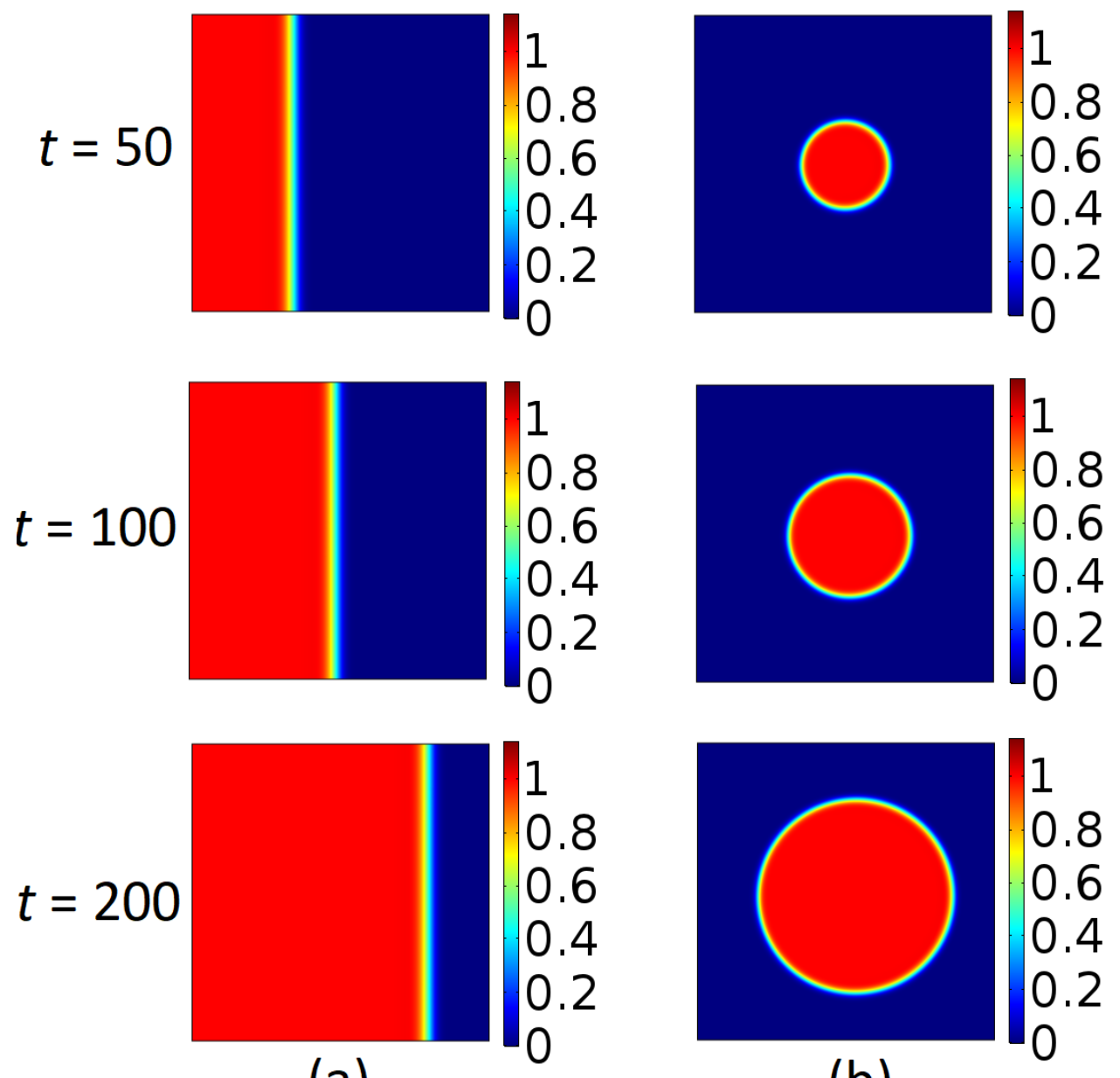

(a)

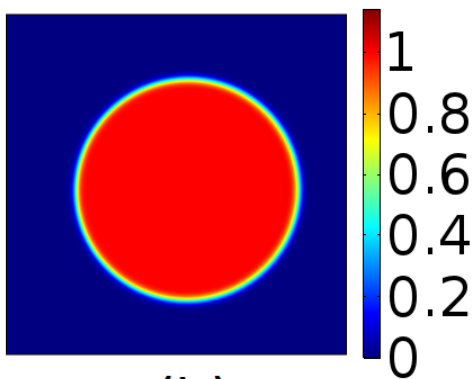

(b)

Fig. 6 Plots of $u_{1}$ in (a) planar and (b) radially symmetric waves at times $t=50,100$, and 200. We take $\delta_{\ell}=A_{\ell}=\mu_{\ell}=$ $\varepsilon_{\ell}=1$ with $a_{11}=a_{22}=1, a_{12}=2, a_{21}=3$, and $b_{\ell j}=a_{\ell j}$. Note that the domain in (a) is taken to be $\Omega=[0,100]^{2}$, whereas the domain in (b) is taken to be $\Omega=[-100,100]^{2}$. 


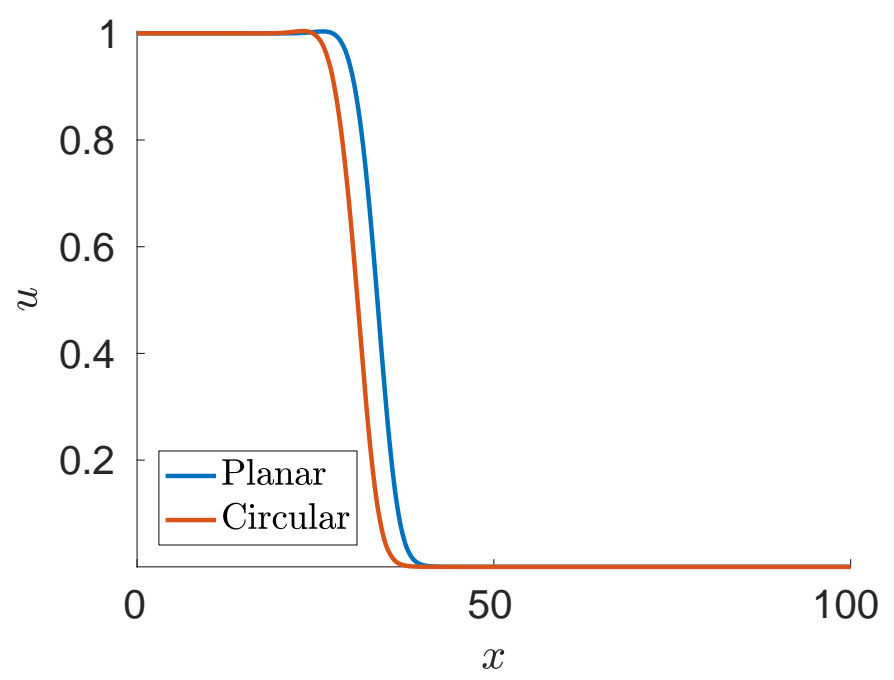

(a)

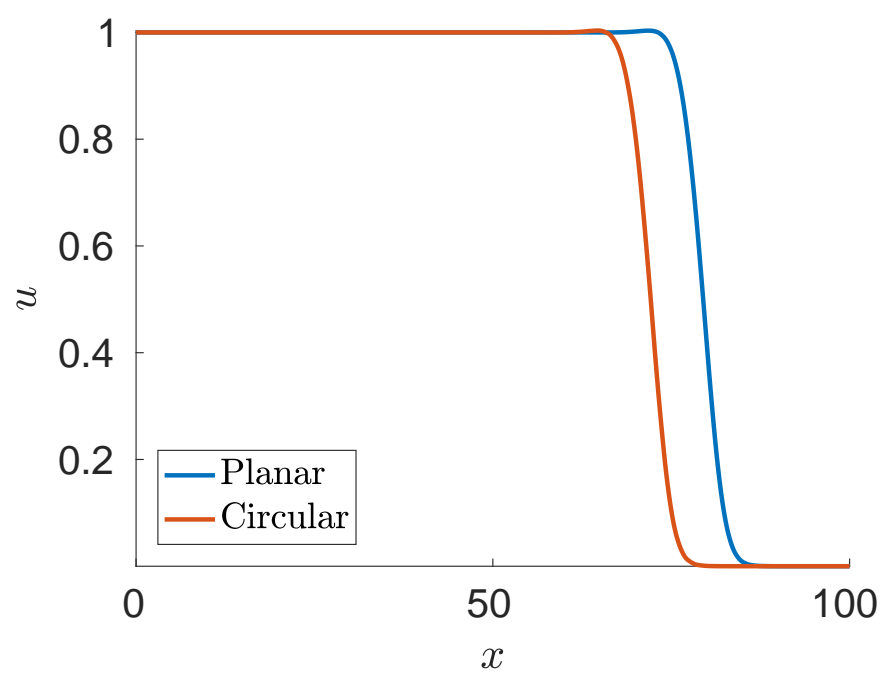

(b)

Fig. 7 Plots of $u_{1}$ in planar and radially symmetric (circular) waves in one dimensional slices from $x_{1}=0$ to $x_{1}=100$ with $x_{2}=0$ fixed. We provide plots at times (a) $t=50$, (b) $t=200$. We take $\delta_{\ell}=A_{\ell}=\mu_{\ell}=\varepsilon_{\ell}=1$ with $a_{11}=a_{22}=1$, $a_{12}=2, a_{21}=3$, and $b_{\ell j}=a_{\ell j}$. 


\section{Wave-Initiated Pattern Formation}

In (Taylor et al, 2020) we derived conditions for the instability of spatially homogeneous states of two and three-species versions of the system (1) leading to patterned states. In the previous sections, we similarly considered traveling waves in one and two-species forms of these models, finding strong evidence that the direction motion of each population can have a nontrivial impact on the emergent wavespeed. While the homogeneous instability analysis is the most commonly performed for a variety of reasons, even Turing (1952) noted that most patterns in the development of organisms do not emerge from homogeneity. Similarly, in ecological contexts, spreading processes leading to colony formation and spatial segregation are common, especially in the wake of invasions (Shigesada et al, 1995; Liebhold and Tobin, 2008; Lockwood et al, 2013). While results about homogeneous instability suggest that the final equilibrium state in such situations cannot be the homogeneous state, linear instability analysis cannot directly be used to infer much about the mode selection of patterns from large perturbations, such as in waveinitiated patterning.

Analytical results on wave-initiated patterns are limited in comparison to those on instability of homogeneous states; see Section 2.6 of Murray (2003) for some discussion of mode selection due to wave-initiated patterning. Myerscough and Murray (1992) used a linear asymptotic analysis to compute the wavelength of a patterned state due to the spreading of a local perturbation in a cell-chemotaxis system. Analytical-numerical approaches have also been employed to estimate mode selection effects behind propogating fronts (Dee and Langer, 1983; Ben-Jacob et al, 1985; Dee and van Saarlos, 1988). Jensen et al (1994) in particular studied the speed of propagation between homogeneous equilibria and Turing structures in the Lengyel-Epstein model of the CDIMA reaction, finding some agreement with experiments at the time. In general, making analytical progress in these kinds of problems is quite difficult, even in the simple case of scalar bistable reaction-diffusion equations. For this reason, we analyze a few interesting scenarios numerically, but leave a more thorough investigation to future work.

In (Taylor et al, 2020) we found that three species were required to obtain patterns with directed motion along fecundity gradients (as in purely diffusive Lotka-Volterra models), though more general directed motion allowed for pattern formation in two species cases. Motivated by these constraints, We study a two competing species example, followed by a three species example. We consider the domain to be the unit square (with no-flux conditions).

We first consider two competing species with directed motion not necessarily proportional to fecundity gradients, motivated by the pattern-formation of such models observed in Figure 4 of (Taylor et al, 2020). We use the initial data,

$$
u_{1}(\mathbf{x}, 0)=u_{1}^{*} S(\mathbf{x}), u_{2}(\mathbf{x}, 0)=u_{2}^{*}(1-S(\mathbf{x})), S(\mathbf{x})=\operatorname{sech}\left(2500\left((x-0.5)^{2}+(y-0.5)^{2}\right)\right)^{2},
$$

where $u_{1}^{*}=A_{1} / a_{11}$ and $u_{2}^{*}=A_{2} / a_{22}$ are the single-species equilibria, representing invasion from a small group of species $u_{1}$ in the center of a large territory of species $u_{2}$. If species $u_{1}$ was more fit than species $u_{2}$, we would anticipate a competitive exclusion invasion comparable to the theoretical wave solutions described in previous sections where the state $\left(u_{1}^{*}, 0\right)$ invades $\left(0, u_{2}^{*}\right)$. Instead, we will choose parameters where these species can stably coexist, but show that inhomogeneous patterning can occur given directed motion which is not along fecundity gradients.

We show examples of this kind of invasion dynamics by plotting transient traveling profiles of $u_{1}$ in Figure 8 for varying values of the coefficients $b_{12}, b_{21}$ representing inter-species directed motion. We remark that for $b_{12}=b_{21}=1$, the directed motion of each species is precisely along fecundity gradients, whereas the other two examples given show stronger inter-species interaction. In this case, as in the purely diffusive case (which has visually identical traveling-wave profiles at each time), the population will eventually spread throughout the entire domain and both species will reach a spatially homogeneous competition equilibrium. For larger inter-species movement parameters, we see less diffuse traveling wave fronts, and these will eventually form spatially inhomogeneous equilibria. We plot these equilibria in Figure 9. As expected, the two populations have a reasonably diffuse boundary between them for $b_{12}=b_{21}=5$, but a sharper boundary for $b_{12}=b_{21}=20$, with the invading population having a smaller overall population, but a denser core than is possible with purely random motion. These patterned states are qualitatively similar to those given in Figure 4 of (Taylor et al, 2020), but we remark that the localized initiation led to an interior localization of $u_{1}$ in this case, whereas random initial data led to colonies with both species adjacent to domain boundaries. This suggests that there are many possible heterogeneous steady states given different initial data, as is often observed in multiple-species patterns.

We next consider three-species pattern formation, as we can compare it directly to the purely diffusive case which also allows for pattern formation behind waves of invasion. The parameters and community 


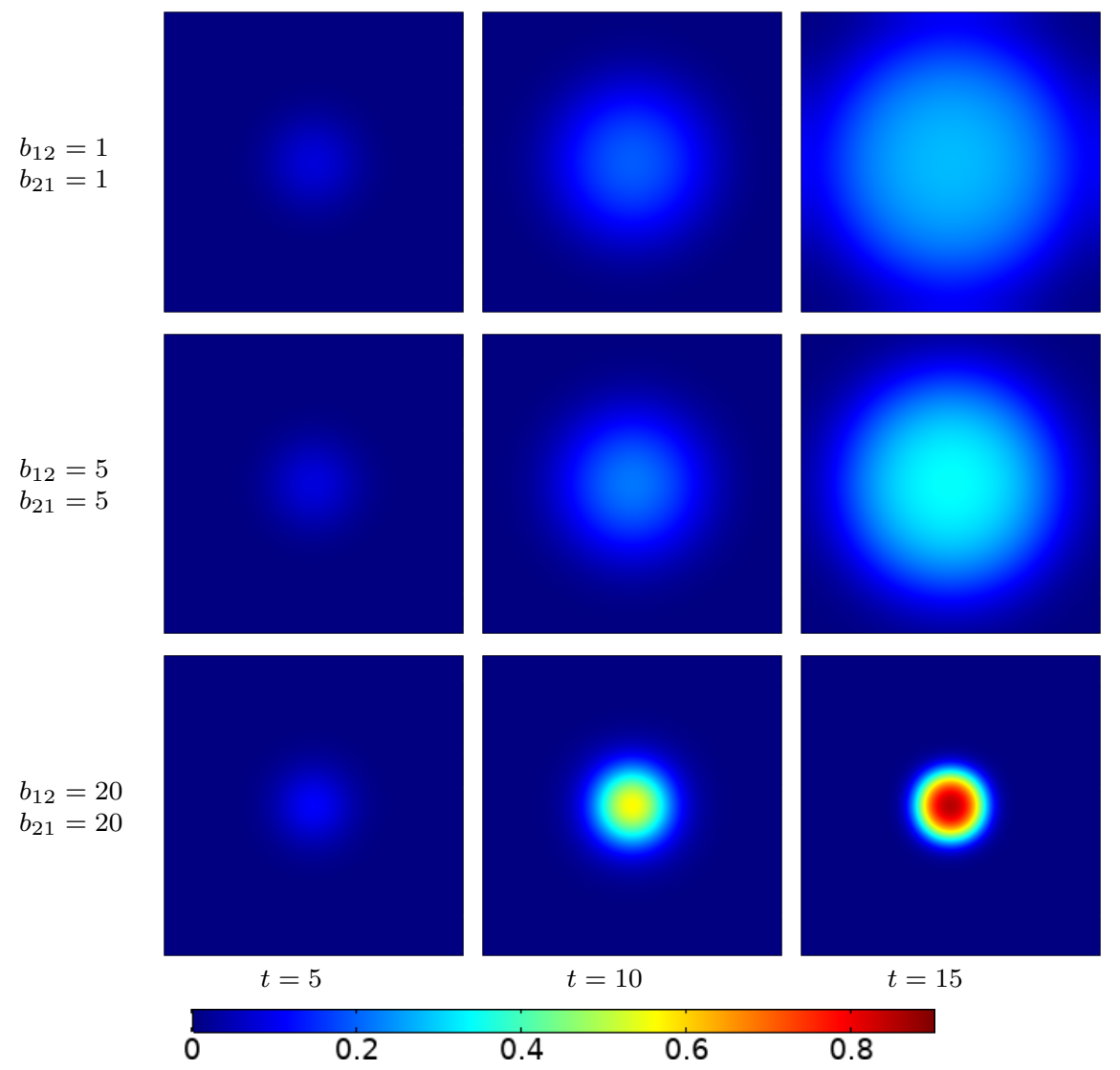

Fig. 8 Plots of the density of $u_{1}$ over time from simulations of two-species competition given by (1) with the initial data (57). We use the parameters $A_{1}=A_{2}=a_{12}=a_{21}=1, a_{11}=a_{22}=2, \delta_{1}=\delta_{2}=10^{-3}, \mu_{1}=\mu_{2}=10^{-2}, \epsilon=10^{-1}$, $b_{11}=b_{22}=2$, and the other advective parameters were varied as labelled.

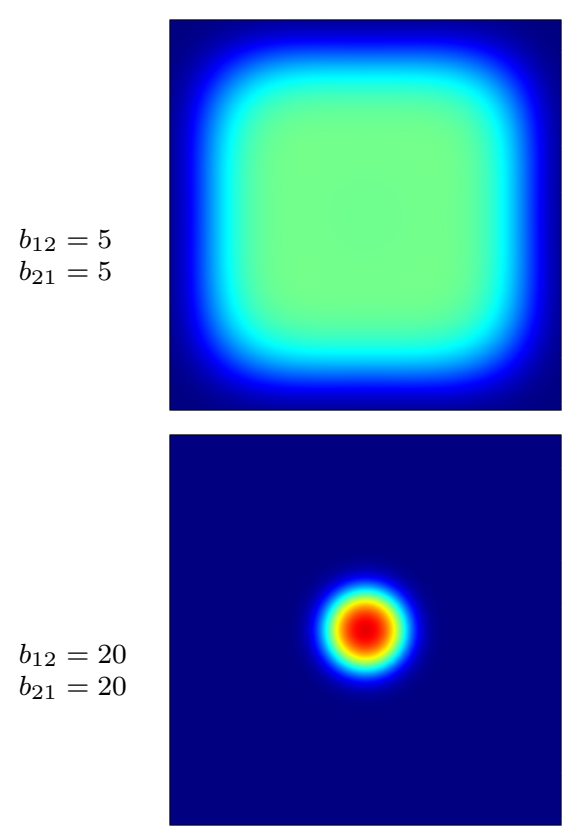

$u_{1}$
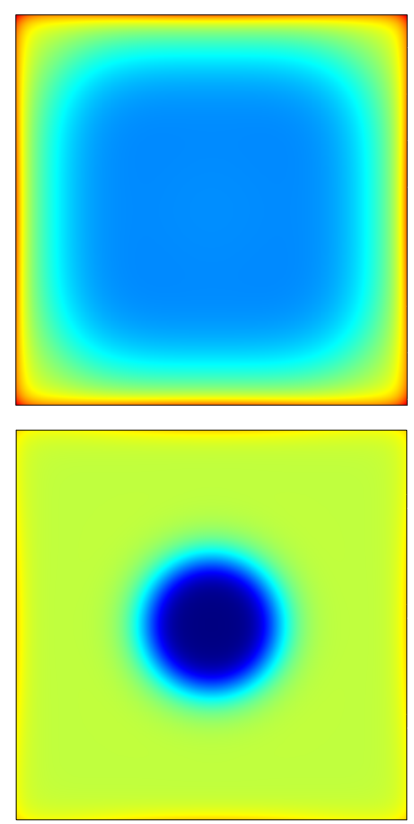

$u_{2}$

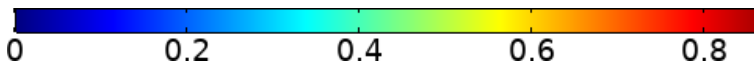

Fig. 9 Final states reached for the populations $u_{1}$ and $u_{2}$ as dwescribed in Figure 8 . Simulations shown at time $t=50$, and they are quantiatively the same over much longer time periods. 
matrix chosen are motivated by Figure 1 in (Taylor et al, 2020), where we have three species competing for resources, and take species $u_{3}$ to also be an intraguild predator which can consume prey $u_{2}$. We consider the domain to be the unit square (with no-flux conditions), and the following initial data:

$$
u_{1}(\mathbf{x}, 0)=u_{1}^{*}(1-S(\mathbf{x})), u_{2}(\mathbf{x}, 0)=u_{2}^{*}(1-S(\mathbf{x})), u_{3}(\mathbf{x}, 0)=u_{3}^{*} S(\mathbf{x}),
$$

with $S(\mathbf{x})$ as defined in $(57), u_{1}^{*}=\left(A_{1} a_{22}-A_{2} a_{12}\right) /\left(a_{11} a_{22}-a_{12} a_{21}\right), u_{2}^{*}=\left(A_{2} a_{11}-A_{1} a_{21}\right) /\left(a_{22} a_{11}-\right.$ $\left.a_{21} a_{12}\right)$, and $u_{3}^{*}=A_{3} / a_{33}$. Such data represent the two purely prey species at their competition equilibria being invaded by a small amount of an omnivorous predator, at its single-species carrying capacity.

We show the transient wave dynamics and final patterned states reached for the predator in Figure 10. The other two species are out-of-phase with the predator, with $u_{1}$ having fairly sharp boundaries and $u_{2}$ being more diffuse, given its much larger diffusion coefficient, $\delta_{2}$. We remark that the first row, giving the purely random motion case, has a mode-selection effect due to the domain boundaries, which was not seen from the random initial perturbation in Figure 1 in (Taylor et al, 2020), which showed a less structured labyrinthine pattern. We then consider directed motion of only one species in each subsequent row. Setting $\mu_{1}=10^{-2}$ in the second row of Figure 10 leads to a sharper traveling wave boundary, and a more curved final predator distribution. Taking $\mu_{2}=10^{-2}$ on the other hand leads to a much more diffuse invasion front and final pattern. Lastly, giving the predator only directed movement $\left(m u_{3}=10^{-2}\right)$ led to a rapid invasion front, and an amalgam of spots and labyrinthine patterns. In all cases except $m u_{1}=10^{-2}$, we observed the original predator colony persist, though in this case the colony grew large enough for a central prey colony to form.

The two-species patterns are somewhat simple, with the broad structure of the original colony remaining the same circular region and some boundary between the two species in their two distinct regions. We anticipate more complicated patterns emerging from distributed but large initial perturbations for example, if multiple competing species invade at different points in a sufficiently large domain. In contrast, the three species simulations demonstrate strong influences of the resulting pattern from the form of the initial wave, as well as its interaction with the domain geometry; note the obvious impact of the square domain in the final patterns of Figure 10 for instance, as well as the impact the invasion wave hitting this barrier has on subsequent interior patterning. We also remark that these waves differ from those in previous sections, as the region behind the invasion front is not a kinetic equilibrium, but a more complicated transient state which is undergoing nonlinear pattern formation; see for instance the column of $t=100$ in Figure 10, where some of the oscillatory pattern formation can be seen behind the wavefront. 

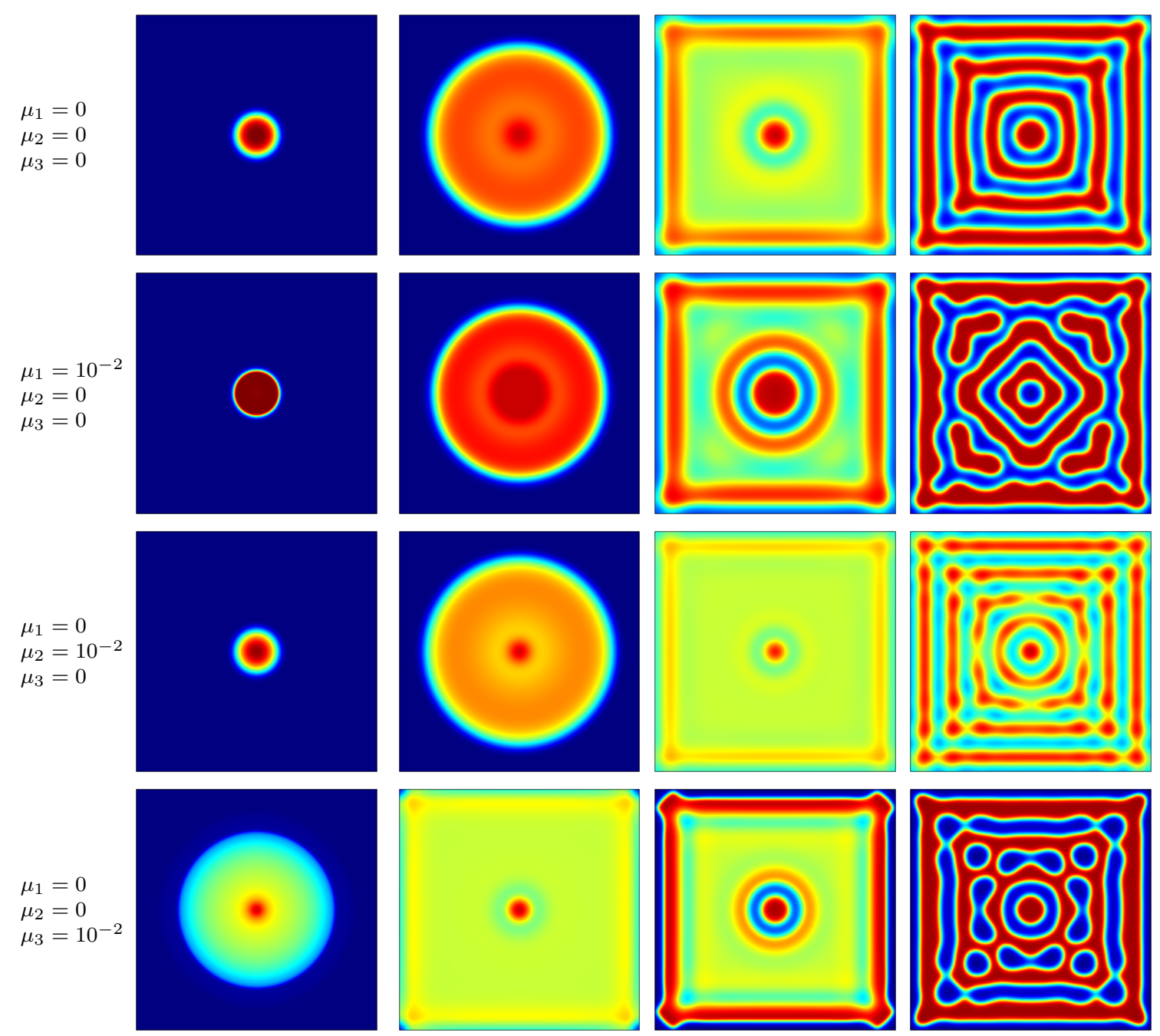

$t=25$

$t=100$

$t=25$

$t=1500$

0.2

0.4

0.6

0.8

Fig. 10 Plots of the density of $u_{3}$ over time from simulations of three-species competition and predation given by (1) with the initial data (58). We use the parameters $A_{1}=A_{2}=A_{3}=1, a_{11}=1.09, a_{12}=0.8, a_{13}=0.81, a_{21}=0.82, a_{22}=1.1$, $a_{23}=0.83, a_{31}=1.68, a_{32}=-85, a_{33}=1.11, \delta_{1}=10^{-6}, \delta_{2}=10^{-3}, \delta_{3}=10^{-5}, \epsilon=10^{-2}$, with directed motion along fecundity gradients so that $F_{\ell}=E_{\ell}$ for $\ell=1,2,3$, and the other advective parameters were varied as labelled. 


\section{Discussion}

Following our analysis of pattern formation in (Taylor et al, 2020), we have studied a variety of traveling wave solutions for the extended model previously studied in Grindrod (1988) and Kurowski et al (2017). The model (1) employs a non-local cross-diffusion mechanism for advection toward resource gradients, and hence our study focused on the role of this type of advection in modifying solutions of traveling wave type found in purely diffusive systems, and observing how these traveling wave structures led to pattern formation distinct from the randomly perturbed homogeneous initial data used in (Taylor et al, 2020).

There have been a variety of wave solution to reaction-diffusion equations and systems with LotkaVolterra kinetics, and in the best known case of Fisher's equation, there exists an exact solution (Ablowitz and Zeppetella, 1979). We extended this exact solution using perturbation theory for small advection, showing analytically that the wavespeed of a wavefront increases with an increase in the advection rate parameter $\mu$, as one might intuitively expect. We then extended the exact solution to a pair of waves for the two-species model. While these exact solutions do not necessarily correspond to the wavespeeds of the asymptotic spreading speed, perturbations of these solutions for small advective parameters showed that generically the advective motion would modify the observed wavespeed. This is a valuable insight as the linear stability analysis for a minimal wavespeed does not anticipate any change due to advective motion.

We then compared our analytical findings with numerical simulations, for both one and two species variants of the model (1). We observed that advection toward resource gradients can increase the wavespeed relative to what is found in purely reaction-diffusion systems, resulting in more rapid wavefront motion for each species, and hence in more effective foraging toward underexploited resources or migration into new regions. In addition to the more commonly studied planar waves, we also provide evidence supporting the existence of radially symmetric waves, which model a population moving out from a small region. For identical parameter values, these radially symmetric wavefronts move more slowly than do the corresponding planar waves, likely due to curvature effects. Still, an increase in the advection rate, $\mu$, increases the wavespeed of radially symmetric waves, as well.

While some of the pattern formation aspects of this model are distinctly different from either a purely diffusive Lotka-Volterra system, or the original model of advection along fecundity gradients presented in Grindrod (1988), broadly we find that the traveling wave character of solutions remains comparable to that in the purely diffusive setting. Linearization predicts the same minimal wavespeed independently of the advection, and while exact solutions of the same form found in the purely diffusive case are no longer possible for our model (1), perturbations due to small advection behave as one would expect by modifying the wavespeed and the front structure only slightly. We do find numerical evidence that larger advection terms, or non-locality of this directed motion, can lead to a substantially changed wavespeed from the purely diffusive case (see Figures 3 and 5). Our simulations in the radial case suggest that the influence of curvature may be enhanced by the non-local nature of the directed motion. Taken together, our analytical and numerical findings suggest that generically these systems may not obey any kind of linear determinacy, where the asymptotic spreading speed is equal to that found from linear stability analysis at the wavefront, though we leave a rigorous investigation of this to future work.

Finally, we investigated wave-initiated pattern formation, motivated by colony formation behind fronts of invasion in various ecological scenarios. We observed a strong impact of both the initial data and the domain geometry, which was not seen in the patterns studied in (Taylor et al, 2020). We showed that the directed motion of species can have profound impacts both on the structure of the traveling fronts, as well as the resulting patterns. While there is more work to do in precisely understanding the mode selection effects, even in the purely diffusive setting, we anticipate that these kinds of waveinitiated patterns are more faithful to real ecological scenarios than random perturbations of homogeneous equilibria.

In addition to the extensions of the model outlined in Taylor et al (2020), there are a number of wavephenomena related aspects which would be valuable to pursue. Rigorously formalizing the arguments made here about the impact of the wavespeed would require a more sophisticated analysis than that presented by Dunbar (1984), due to the higher-dimensionality of the system, the coupling of parabolic and elliptic equations, and the nonlinear advection terms. Similarly, while we conjecture that linear determinacy never holds for nonzero advection, rigorously showing this or classifying under what parameter restrictions it might still hold remains to be done, though there has been much recent progress in the purely diffusive Lotka-Volterra setting (Alhasanat and Ou, 2019; Girardin and Lam, 2019). More relevant for ecological analysis would be to extend the kinds of directed motion to include other kinds of taxis and cues between species, as recently studied in pure dispersal models by Potts and Lewis (2019). 
Allee effects have been shown to have profound impacts on the structure of traveling waves and invasive fronts in populations (Wang and Kot, 2001; Liebhold and Tobin, 2008; Roques et al, 2012), and these cannot be captured by the linear Lotka-Volterra functional responses we have considered. Stochasticity at the wavefront can also have profound impacts on the colony formation behind an invasion (Liebhold and Tobin, 2008). Therefore, extending these models to more realistic functional responses would also be worthwhile, as some of our results may change substantially.

\section{References}

Ablowitz MJ, Zeppetella A (1979) Explicit solutions of Fisher's equation for a special wave speed. Bulletin of Mathematical Biology 41(6):835-840

Alhasanat A, Ou C (2019). Minimal-speed selection of traveling waves to the Lotka-Volterra competition model. Journal of Differential Equations, 266(11):7357-7378

Al-Kiffai A, Crooks E (2016) Lack of symmetry in linear determinacy due to convective effects in reactiondiffusion-convection problems. Tamkang Journal of Mathematics 47(1)

Aronson DG, Weinberger HF (1978). Multidimensional nonlinear diffusion arising in population genetics. Advances in Mathematics, 30(1), 33-76

Ben-Jacob E, Brand H, Dee G, Kramer L, Langer JS (1985) Pattern propagation in nonlinear dissipative systems. Physica D: Nonlinear Phenomena 14(3):348-364

Berestycki H (2002) The influence of advection on the propagation of fronts in reaction-diffusion equations. In: Nonlinear PDE's in Condensed Matter and Reactive Flows, Springer, pp 11-48

Berestycki H, Hamel F (2007) Generalized travelling waves for reaction-diffusion equations. Contemporary Mathematics 446:101-124

Bertsch M, Gurtin ME, Hilhorst D, Peletier L (1985) On interacting populations that disperse to avoid crowding: preservation of segregation. Journal of Mathematical Biology 23(1):1-13

Castillo-Chavez C, Li B, Wang H (2013) Some recent developments on linear determinacy. Mathematical Biosciences \& Engineering, 10(5\& 6):1419-1436

del Castillo-Negrete D, Carreras B, Lynch V (2002) Front propagation and segregation in a reactiondiffusion model with cross-diffusion. Physica D: Nonlinear Phenomena 168:45-60

Chen X, Hambrock R, Lou Y (2008) Evolution of conditional dispersal: a reaction-diffusion-advection model. Journal of Mathematical Biology 57(3):361-386

Dee G, Langer JS (1983) Propagating pattern selection. Physical Review Letters 50(6):383

Dee GT, van Saarloos W (1988) Bistable systems with propagating fronts leading to pattern formation. Physical review letters 60(25):2641

Dormand JR, Prince PJ (1980) A family of embedded Runge-Kutta formulae. Journal of computational and applied mathematics 6(1):19-26

Dunbar SR (1983) Travelling wave solutions of diffusive Lotka-Volterra equations. Journal of Mathematical Biology 17(1):11-32

Dunbar SR (1984) Traveling wave solutions of diffusive Lotka-Volterra equations: a heteroclinic connection in $\mathbb{R}^{4}$. Transactions of the American Mathematical Society pp 557-594

Fisher RA (1937) The wave of advance of advantageous genes. Annals of Eugenics 7(4):355-369

Gambino G, Lombardo MC, Sammartino M (2012) Turing instability and traveling fronts for a nonlinear reaction-diffusion system with cross-diffusion. Mathematics and Computers in Simulation 82(6):11121132

Gambino G, Lombardo M, Sammartino M (2013) Pattern formation driven by cross-diffusion in a $2 \mathrm{~d}$ domain. Nonlinear Analysis: Real World Applications 14(3):1755-1779

Girardin L, Nadin G (2015). Travelling waves for diffusive and strongly competitive systems: relative motility and invasion speed. European Journal of Applied Mathematics, 26(4):521-534

Girardin L (2018). Non-cooperative Fisher-KPP systems: asymptotic behavior of traveling waves. Mathematical Models and Methods in Applied Sciences, 28(06):1067-1104

Girardin L, Lam KY (2019) Invasion of open space by two competitors: spreading properties of monostable two-species competition-diffusion systems. Proceedings of the London Mathematical Society 119(5):1279-1335

Goriely A (1996) Integrability, partial integrability, and nonintegrability for systems of ordinary differential equations. Journal of Mathematical Physics 37(4):1871-1893

Grindrod P (1988) Models of individual aggregation or clustering in single and multi-species communities. Journal of Mathematical Biology 26(6):651-660 
Grindrod P (1991) Patterns and waves: The theory and applications of reaction-diffusion equations. Oxford University Press, USA

Hambrock R, Lou Y (2009) The evolution of conditional dispersal strategies in spatially heterogeneous habitats. Bulletin of Mathematical Biology 71(8):1793

Hearns J, Van Gorder RA, Choudhury SR (2012) Painlevé test, integrability, and exact solutions for density-dependent reaction-diffusion equations with polynomial reaction functions. Applied Mathematics and Computation 219(6):3055-3064

Hillen T, Painter KJ (2009) A user's guide to PDE models for chemotaxis. Journal of Mathematical Biology 58(1-2):183

Horstmann D (2007) Remarks on some Lotka-Volterra type cross-diffusion models. Nonlinear Analysis: Real World Applications 8(1):90-117

Hosono Y (1998) The minimal speed of traveling fronts for a diffusive Lotka-Volterra competition model. Bulletin of Mathematical Biology 60(3):435-448

Huang W, Han M (2011) Non-linear determinacy of minimum wave speed for a Lotka-Volterra competition model. Journal of Differential Equations, 251(6):1549-1561

Gurtin ME, MacCamy RC (1977) On the diffusion of biological populations. Mathematical Biosciences 33(1-2):35-49

Ibrahim H, Nasreddine E (2017) Traveling waves for a model of individual clustering with logistic growth rate. Journal of Mathematical Physics 58(8):081505

Jensen O, Pannbacker VO, Mosekilde E, Dewel G, Borckmans P. (1994) Localized structures and front propagation in the Lengyel-Epstein model. Physical Review E 50(2):736

Jones CK (1983) Spherically symmetric solutions of a reaction-diffusion equation. Journal of Differential Equations 49(1):142-169

Kareiva P, Odell G (1987). Swarms of predators exhibit "preytaxis" if individual predators use arearestricted search. The American Naturalist 130(2):233-270.

Keener JP (1986) A geometrical theory for spiral waves in excitable media. SIAM Journal on Applied Mathematics 46(6):1039-1056

Keener JP (1991) An eikonal-curvature equation for action potential propagation in myocardium. Journal of mathematical biology 29(7):629-651

Kiselev A, Ryzhik L (2001) Enhancement of the traveling front speeds in reaction-diffusion equations with advection. Annales de l'Institut Henri Poincaré (C) Non Linear Analysis 18(3):309-358

Kurowski L, Krause AL, Mizuguchi H, Grindrod P, Van Gorder RA (2017) Two-species migration and clustering in two-dimensional domains. Bulletin of Mathematical Biology 79(10):2302-2333

Kuznetsov YA, Antonovsky MY, Biktashev V, Aponina E (1994) A cross-diffusion model of forest boundary dynamics. Journal of Mathematical Biology 32(3):219-232

Lewis MA, Petrovskii SV, Potts JR (2016) The mathematics behind biological invasions. Springer, Berlin

Lewis MA, Li B, Weinberger HF (2002) Spreading speed and linear determinacy for two-species competition models. Journal of mathematical biology, 45(3):219-233

Li B, Weinberger HF, Lewis MA (2005) Spreading speeds as slowest wave speeds for cooperative systems. Mathematical biosciences, 196(1):82-98

Liebhold AM, Tobin PC (2008) Population ecology of insect invasions and their management. Annu. Rev. Entomol. 53 387-408

Lockwood JL, Hoopes MF, Marchetti MP (2013) Invasion ecology. John Wiley \& Sons.

Miller PD (1997) Nonmonotone waves in a three species reaction-diffusion model. Methods and Applications of Analysis 4(3):261-282

Murray JD (2003) Mathematical Biology II: Spatial Models and Biomedical Applications. Springer, New York

Myerscough MR, Murray JD (1992) Analysis of propagating pattern in a chemotaxis system. Bulletin of Mathematical Biology, 54(1):77-94

Okubo A, Maini PK, Williamson MH, Murray JD (1989) On the spatial spread of the grey squirrel in Britain. Proceedings of the Royal Society of London B Biological Sciences 238(1291):113-125

Pettet G, McElwain D, Norbury J (2000) Lotka-Volterra equations with chemotaxis: Walls, barriers and travelling waves. Mathematical Medicine and Biology: A Journal of the IMA 17(4):395-413

Potts JR, Lewis MA (2019) Spatial memory and taxis-driven pattern formation in model ecosystems. Bulletin of mathematical biology 81:2725-2747

Ramani A, Grammaticos B, Bountis T (1989) The Painlevé property and singularity analysis of integrable and non-integrable systems. Physics Reports 180(3):159-245 
Roques L, Garnier J, Hamel F, Klein EK (2012) Allee effect promotes diversity in traveling waves of colonization. Proceedings of the National Academy of Sciences 109(23):8828-8833

Roussier V (2004) Stability of radially symmetric travelling waves in reaction-diffusion equations. Annales de l'IHP Analyse non linéaire 21(3):341-379

Russo M, Van Gorder RA, Choudhury SR (2013) Painlevé property and exact solutions for a nonlinear wave equation with generalized power-law nonlinearities. Communications in Nonlinear Science and Numerical Simulation 18(7):1623-1634

Satnoianu RA (2003) Coexistence of stationary and traveling waves in reaction-diffusion-advection systems. Physical Review E 68(3):032101

Sherratt JA (1993) Cellular growth control and travelling waves of cancer. SIAM Journal on Applied Mathematics 53(6):1713-1730

Shigesada N, Kawasaki K, Takeda Y (1995) Modeling stratified diffusion in biological invasions. The American Naturalist, 146(2):229-251

Strobl MAR, Krause AL, Damaghi M, Gillies R, Anderson ARA, Maini PK (2020) Mix \& Match: Phenotypic coexistence as a key facilitator of solid tumour invasion. Bulletin of Mathematical Biology $15(2)$

Taylor NP, Kim H, Krause AL, Van Gorder RA (2020) A non-local cross-diffusion model of population dynamics I: Emergent spatial and spatiotemporal patterns. Bulletin of Mathematical Biology

Turing AM (1952) The chemical basis of morphogenesis. Philosophical Transactions of the Royal Society of London Series B, Biological Sciences 237(641):37-72

Volpert V, Petrovskii S (2009) Reaction-diffusion waves in biology. Physics of life reviews 6(4):267-310

Wang MH, Kot M (2001) Speeds of invasion in a model with strong or weak Allee effects. Mathematical biosciences 171(1):83-97

Wang ZA (2013) Mathematics of traveling waves in chemotaxis-Review paper-. Discrete \& Continuous Dynamical Systems-B 18(3):601-641

Weiss J (1983) The Painlevé property for partial differential equations. II: Bäcklund transformation, Lax pairs, and the Schwarzian derivative. Journal of Mathematical Physics 24(6):1405-1413

Weiss J, Tabor M, Carnevale G (1983) The Painlevé property for partial differential equations. Journal of Mathematical Physics 24(3):522-526

White A, Lurz PW, Jones HE, Boots M, Bryce J, Tonkin M, Ramoo K, Bamforth L, Jarrott A (2015) The use of mathematical models in red squirrel conservation: Assessing the threat from grey invasion and disease to the Fleet basin stronghold. Red Squirrels Ecology, Conservation Management in Europe; Shuttleworth C, Lurz PWW, Hayward MW, Eds, pp.265-279

Wu YP (1995) Traveling waves for a class of cross-diffusion systems with small parameters. Journal of Differential Equations 123(1):1-34 